\title{
Uganda: Third Review Under the Policy Support Instrument and Request for Waiver and Modification of Assessment Criteria-Staff Report; Press Release on the Executive Board Discussion; and Statement by the Executive Director for Uganda
}

In the context of the third review under the Policy Support Instrument and request for a waiver and modification of assessment criteria, the following documents have been released and are included in this package:

- $\quad$ The staff report for the Third Review Under the Policy Support Instrument and Request for Waiver and Modification of Assessment Criteria, prepared by a staff team of the IMF, following discussions that ended on May 27, 2008, with the officials of Uganda on economic developments and policies. Based on information available at the time of these discussions, the staff report was completed on June 26,2008. The views expressed in the staff report are those of the staff team and do not necessarily reflect the views of the Executive Board of the IMF.

- $\quad$ A Press Release summarizing the views of the Executive Board as expressed during its July 9, 2008 discussion of the staff report that completed the review.

- $\quad$ A statement by the Executive Director for Uganda.

The documents listed below have been or will be separately released.

Letter of Intent sent to the IMF by the authorities of Uganda*

Memorandum of Economic and Financial Policies by the authorities of Uganda*

Technical Memorandum of Understanding*

*Also included in Staff Report

The policy of publication of staff reports and other documents allows for the deletion of marketsensitive information.

Copies of this report are available to the public from

International Monetary Fund • Publication Services

$70019^{\text {th }}$ Street, N.W. • Washington, D.C. 20431

Telephone: (202) 623-7430 • Telefax: (202) 623-7201

E-mail: publications@imf.org • Internet: http://www.imf.org

Price: $\$ 18.00$ a copy

\section{International Monetary Fund Washington, D.C.}



INTERNATIONAL MONETARY FUND

UGANDA

Third Review Under the Policy Support Instrument and Request for Waiver and Modification of Assessment Criteria

Prepared by the African Department

(In consultation with other departments)

Approved by Thomas Krueger and Mark Plant

June 25, 2008

- Summary. This report recommends completion of the third review under the Policy Support Instrument (PSI), which was approved by the Executive Board on December 15, 2006. It notes the economy's ability to weather shocks and discusses changes to the authorities' macroeconomic policies.

- $\quad$ Participants. The mission team comprised Messrs. Corker (head), Selassie (senior resident representative), Gershenson, Saenz (all AFR), Ms. Brunschwig (PDR), and Mr. Jonas (FAD). It visited Kampala on March 10-20 and May 19-27 and met with Finance Minister Suruma, Bank of Uganda Governor Tumusiime Mutebile, other officials, and representatives of the international community. Mr. Mwanawina, advisor to Executive Director, participated in the discussions. 


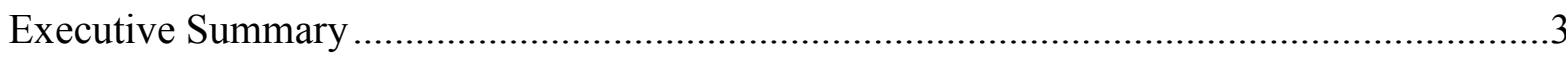

I. Good Performance and New Challenges...............................................................4

II. Third Review—Broadly On Track.......................................................................

III. Outlook: Strong Growth Supported by Higher Public Investment .................................

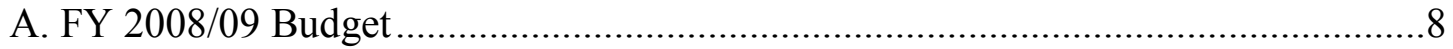

B. Monetary Policy and Financial Sector ............................................................ 10

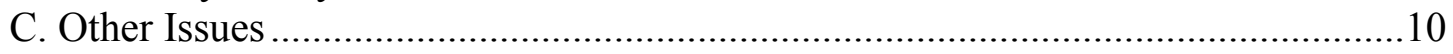

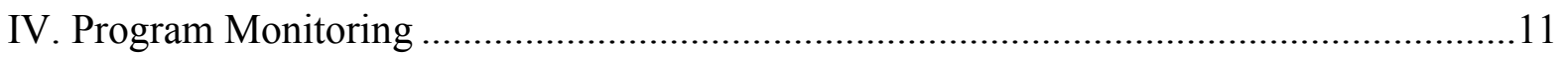

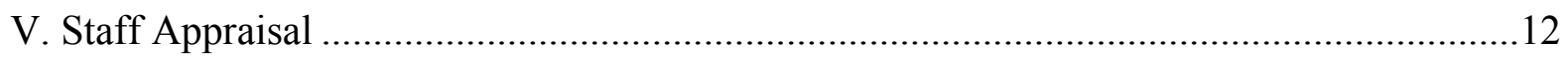

Box

Revisions to the National Account ..............................................................................

Tables

1. Selected Economic and Financial Indicators, 2005/06-2008/09..............................13

2. Fiscal Operations of the Central Government, 2005/06-2008/09 ............................14

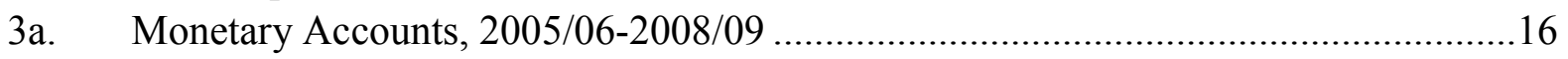

3b. Quarterly Monetary Accounts 2006/07-2008/09 ..................................................17

4. Balance of Payments, 2005/06-2010/11 ................................................................ 18

5. Medium-Term Macroeconomic Framework, 2005/06-2009/10 ................................19

6. Quantitative Assessment Criteria and Indicative Targets for end-December 2007

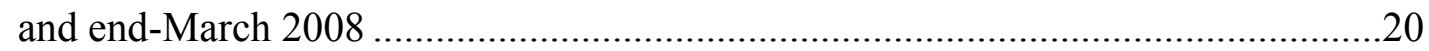

7. Structural Assessment Criteria and Benchmarks .............................................21

8. Selected Banking Sector Information, June 2004-December 2007 ..........................23

9. Millennium Development Goals ....................................................................24

Appendices

I. Letter of Intent ...............................................................................................25

Attachment I. Memorandum of Economic and Financial Policies ......................27

Attachment II. Technical Memorandum of Understanding .................................36 


\section{Executive Summary}

Staff recommends the completion of the third review. All end-December 2007 assessment criteria were met, and staff supports the authorities' request for waivers of nonobservance of four structural assessment criteria for end-May and end-June 2008, relating to the national identity card system, nonbank regulation, the Integrated Personnel and Payroll System, and budget allocation for arrears clearance. Nonobservance was largely due to changes in program design in light of new information and reassessment of costs and priorities that do not compromise the integrity of the program. Macroeconomic performance remains strong and the growth outlook remains positive.

Starting in 2008/09, the authorities plan to scale up domestic investment to alleviate the pressing infrastructure bottlenecks. Care needs to be taken to ensure that money is spent well and that the concomitant real decline in current spending does not result in new domestic arrears.

The Bank of Uganda (BoU) faces the challenge of bringing inflation back below 5 percent. An increase in domestic inflation reflects mainly the sharp pick up in global fuel and food prices. The BoU has appropriately accommodated the first round effects on local prices and is committed to preventing any spill over into sustained higher inflation.

Large foreign exchange inflows continue to pose an additional challenge to monetary policy. Because of concerns about a further shilling appreciation and loss of competitiveness, the BoU has placed emphasis on open market operation to sterilize the liquidity effects. However, this has entailed sizable fiscal costs.

Despite the more demanding external environment, program risks remain manageable. High foreign exchange reserves and large reliance on local food production make Uganda less vulnerable to higher food and oil prices. Revenue performance continues to improve, and prudent expenditure management should assure continued fiscal discipline. However, repeated failure to arrest the growth of domestic arrears suggests the need for a thorough reexamination of the integrated financial management system (IFMS), and the appropriateness of budgetary allocations. Uganda is assessed to be at low risk of debt distress. 


\section{Good Performance ANd New Challenges}

1. Uganda's economy continues to thrive. Playing off improved regional stability and a construction boom (partly related to last year's Commonwealth Heads of Government Meeting, CHOGM), newly revised national accounts estimates (see Box) show the economy expanded by nearly 10 percent a year over the last three years. Demand for Ugandan goods from South Sudan and the Democratic Republic of the Congo, in particular, has been strong.

\section{Box. Revisions to the National Accounts}

The Uganda Bureau of Statistics recently released revised estimates of the national accounts. The exercise entailed two changes: improved methodology to estimate sectoral outputs and a rebasing of all series to 2002. As a result, most sectors are now better measured and those that have been growing rapidly in recent years now have weights that better reflect their share in economic activity.

The new estimates show nominal GDP to be some 5 percent higher in 2006/07 than previously reported and real GDP growth 3 percentage points higher. The industrial (construction) and services (telecoms, financial services) sectors contributed more to growth as a result of their increased weights and better measurement. The pick-up in growth from around 2005/06 corresponds roughly to the time when reconstruction activity in South Sudan (and to a lesser degree the Democratic Republic of Congo) took off.

Estimates of real GDP growth, 2003/04 - 2007/08

\begin{tabular}{|c|c|c|c|c|c|c|c|}
\hline & \multirow[t]{2}{*}{$2003 / 04$} & \multirow[t]{2}{*}{$2004 / 05$} & \multirow[t]{2}{*}{$2005 / 06$} & \multirow[t]{2}{*}{$2006 / 07$} & \multirow{2}{*}{ 2007/08 1/ } & \multicolumn{2}{|c|}{ Averages } \\
\hline & & & & & & Last 5 years & Last 3 years \\
\hline \multicolumn{8}{|l|}{ Revised } \\
\hline Market price & 6.8 & 6.3 & 10.8 & 7.9 & 9.8 & 8.3 & 9.5 \\
\hline Factor cost & 6.4 & 6.6 & 10.3 & 7.4 & 9.0 & 7.8 & 8.9 \\
\hline \multicolumn{8}{|l|}{ Original } \\
\hline Market price & 5.4 & 6.8 & 5.1 & 6.7 & 6.9 & 6.2 & 6.2 \\
\hline Factot cost & 5.3 & 6.5 & 5.0 & 6.4 & 6.9 & 6.0 & 6.1 \\
\hline
\end{tabular}

Source: Ugandan authorities.

1/ Projections

The new data imply a lower level and slower rate of increase in the tax-to-GDP ratio. Likewise, the level of money velocity is raised and its rate of decline appears slower. Pending further analysis in the context of the upcoming Article IV discussions, the revised trends in these variables as well as the stronger underlying growth momentum of the economy have been provisionally factored into program estimates and projections. 
The newly-founded, albeit precarious, stability in Northern Uganda has also started to contribute to growth. The civil unrest in Kenya-which erupted in January and temporarily blocked Uganda's main outlet to the sea-appears to have had only a short-lived impact on economic activity, with roads reopening quickly. Shortages in Kenya as well as the disruption to trade routes, did however contribute to higher prices and transportation costs.
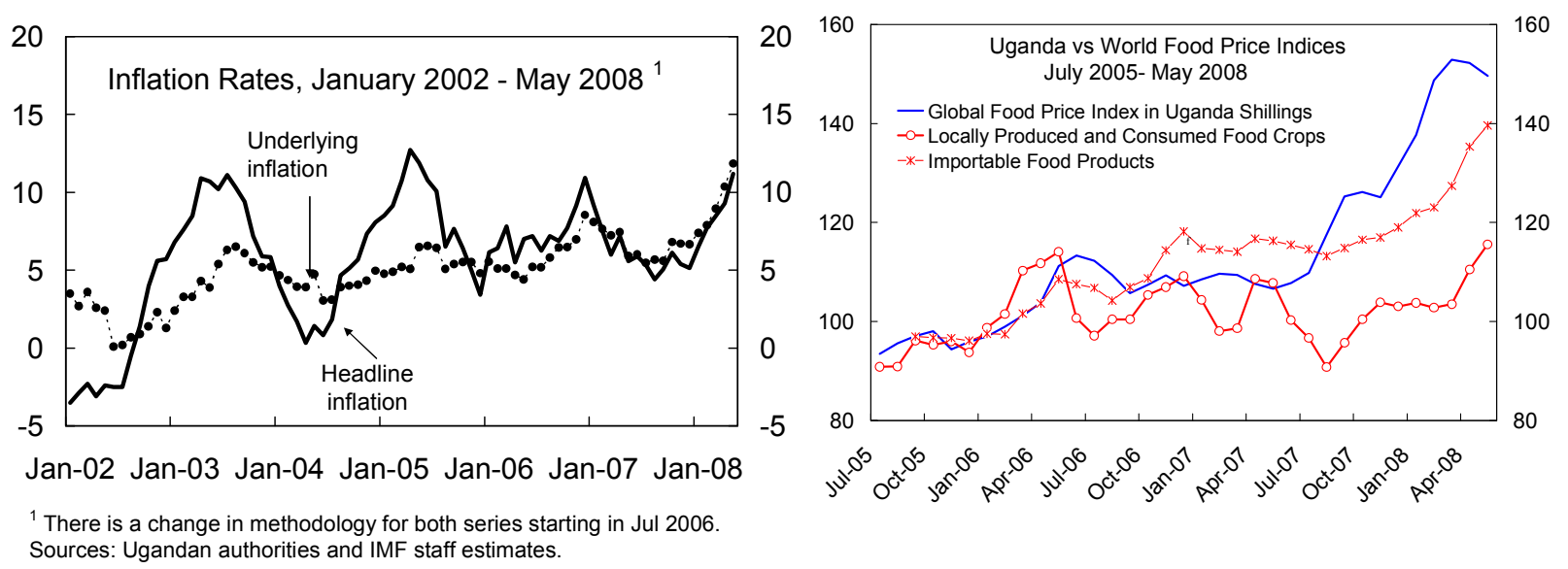

2. Rising world food and fuel prices have, however, in recent months pushed core inflation $^{1}$ into the double digits for the first time since the mid-1990s. In April, core inflation increased to nearly 11 percent, with some 2.5 percentage point directly attributable to its tradable food component. At the same time, reflecting relatively good recent harvests, the prices of locally produced and consumed staple foods have been broadly stable.

3. The already-sizeable foreign exchange inflows intensified in 2007/08. Much of the inflows were sterilized through open market operations, allowing the shilling to appreciate only modestly against U.S. dollar, but depreciate against the Euro and in effective terms. The use of government securities to mop-up excess liquidity raised the budgetary costs of open market operations by a further $1 / 4$ percentage point of GDP in 2007/08.

\footnotetext{
${ }^{1}$ Core inflation excludes volatile energy and locally produced food, but includes tradable food (such as rice and maize), and items affected by fuel prices (such as transportation).
} 

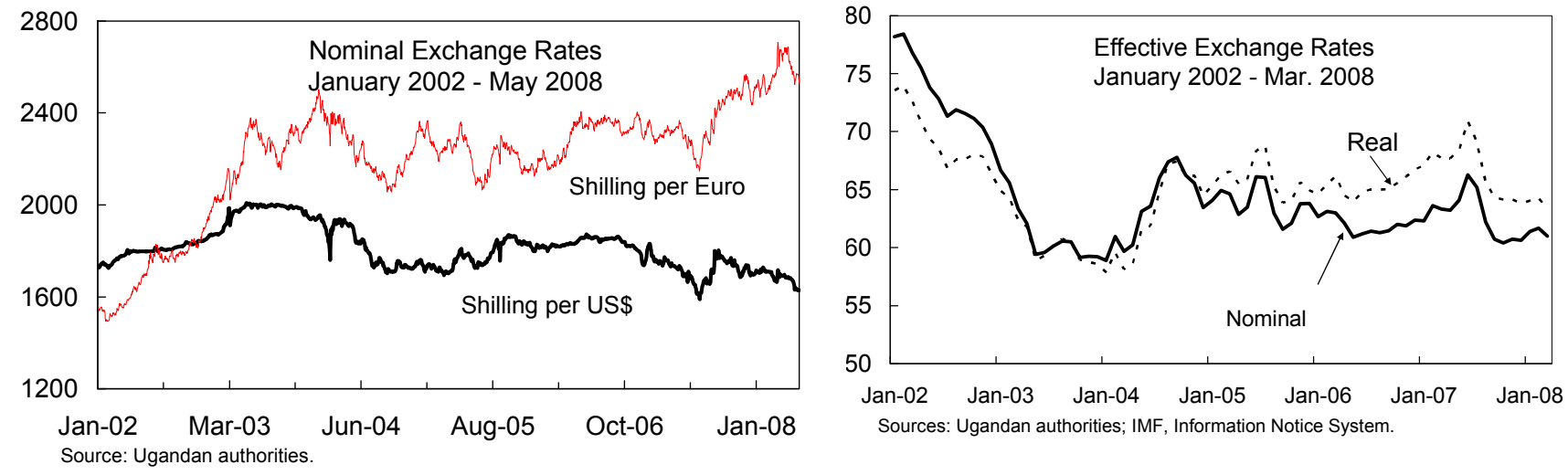

\section{THIRD REVIEW-BROADLY ON TRACK}

4. All assessment criteria through end-December 2007 were met (Tables 6 and 7):

- Greater-than-budgeted spending related to CHOGM, purchase of a new presidential jet, and the costs of natural disasters - floods in North and East, Ebola outbreak in the West - have been offset by across-the-board expenditure cuts in non-priority areas and strong revenue performance. In addition, earlier-than-programmed repayment of the Bujagali bridge loan and technical delays in budget execution contributed to a substantial overperformance of the assessment criterion on net credit to government.

- $\quad$ Strong foreign exchange inflows and the authorities' concern for exporters have driven the stock of international reserves of the BOU well above the program floor. At the same time, the indicative target on base money target was exceeded by 5.4 percent because these inflows were sterilized only partially in order to avoid a spike in interest rates. ${ }^{2}$ The ceiling on net domestic assets of the BOU was comfortably observed.

- $\quad$ Financing for the Bujagali hydropower project was secured in December 2007. The related non-concessional guarantee, at US\$115 million, is well within the program ceiling.

- $\quad$ The government's debt strategy was published in December 2007.

\footnotetext{
${ }^{2}$ The authorities aim to keep base money within \pm 5 percent of the indicative target. The range was slightly exceeded at end-December 2007 due to the lack of timely accurate data on the reserves held by several new banks that started operations late last year. The data have since been revised.
} 
- $\quad$ The indicative target on the stock of domestic budgetary arrears is not being observed. Corrected estimates for 2006/07 show that, contrary to what was reported in Country Report 08/4, the indicative ceiling was exceeded by a large margin. ${ }^{3}$ Partly due to a higher stock of arrears at the beginning of the fiscal year, the December 2007 target is expected to have been exceeded by at least 0.3 percent of GDP, although precise estimates will only be available with further lag.

- $\quad$ Even though the required amount was released by the Ministry of Finance, the indicative target on poverty-related spending was not observed due to delays in recruitment and compliance by the spending agencies.

\section{Four structural assessment criteria with end-May and end-June test dates will} not be met (Table 7). This is largely due to changes in program design in light of new information and reassessment of costs and priorities that do not compromise the integrity of the program:

- A tender to select the provider for the National ID system is being delayed due to cost considerations; for the time being the authorities intend to enhance existing databases instead (MEFP, $₫ 14$ );

- In lieu of a single regulatory body for the non-bank financial sector, the authorities have opted for specialized regulatory agencies (MEFP, $\mathbb{\| 1 2}$ );

- The authorities now expect the new Integrated Personnel and Payroll System to be rolled out to the relevant Ministries by end-January 2009 rather than end-May 2008 due to delays in the procurement process (MEFP, $₫ 8$ ).

- The authorities have allocated U Sh 300 billion in the 2008/09 budget for arrears clearance. However, the authorities would like to use this allocation to clear all verified arrears rather than just group (A) arrears. Clearance of pension arrears remains a priority.

\section{OUtlook: STRONG Growth Supported by Higher Public InVESTMENT}

6. The immediate macroeconomic outlook is positive. Economic growth is projected to forge ahead at 8 percent as ongoing reconstruction activity in neighboring countries continues to provide an outlet for Ugandan goods. The authorities pointed to encouraging signs of companies ramping up investment to exploit this opportunity. They attributed the

\footnotetext{
${ }^{3}$ A correction in the stock of arrears for 2006/07 reported in Country Report 08/4 is necessary as (i) that number erroneously included only arrears accumulated in 2006/07, and (ii) the 2006/07 arrears estimate has since been revised upwards by the authorities.
} 
resilience of the economy in the face of recent shocks to flexible markets and dynamism of the private sector. With Uganda self-sufficient in food production - and even a net exporter in some years - the authorities felt, and staff concurred, that the country is well-positioned to benefit from higher global food prices. The projected increase in the oil import bill (of some one percent of GDP) is expected to be comfortably covered by the continued strong foreign exchange inflows.

7. Following the strong fiscal consolidation of recent years, the authorities plan to scale up domestic investment to alleviate the pressing infrastructure bottlenecks that pose a risk to medium-term growth. The cornerstone is a major initiative to upgrade the key transport corridors, some sections of which are already being financed by donors, at a cost of some 4 percent of GDP over the next three years. This investment is to be financed through a combination of cuts in current spending and higher domestic borrowing, and is consistent with the priorities set out in the government's debt strategy. A high share of imported goods and services in road construction would help minimize the ensuing crowding out of the private sector, and Uganda's more-than-comfortable level of foreign exchange reserves would provide cover for the additional imports required. The World Bank agrees on the need to scale up investment in roads and will work closely with the authorities to facilitate implementation. The authorities are also looking to develop Karuma, another substantial hydropower project, to augment Uganda's electricity supply.

\section{A. FY 2008/09 Budget}

8. The 2008/09 budget ushers in a significant shift in fiscal policy. With development spending slated to increase from 3 to $5 \frac{1}{2}$ percent of GDP, the recent era of fiscal consolidation will cease. Staff welcomed the reorientation towards development spending and estimated that it could be accommodated with the macroeconomic program. The main question was whether there was adequate absorption capacity to ensure value for money. The authorities noted that recent initiatives to restructure agriculture extension service programs and the creation of the Uganda National Roads Authority, among others, would enhance absorption capacity. They are also considering "turnkey" contracts in the roads sector, consistent with national procurement regulations to speed-up project implementation (MEFP, ๆ5).

\section{The increased spending is set to be partly financed through the reallocation of resources from current primary spending and higher domestic borrowing.}

a. Current primary spending is projected to decline by close to $1 \frac{1 / 4}{4}$ percent of GDP (see text table). The authorities discounted the possibility that this compression could result in a further build-up of domestic arrears (see below). They felt that there was significant scope to curb wasteful public spending, and pointed to their success in 2007/08 in scaling back non-priority spending. 
Uganda: Domestic Primary Balance 2003/04-2008/09

\begin{tabular}{|c|c|c|c|c|c|c|}
\hline & $2003 / 04$ & $2004 / 05$ & $2005 / 06$ & 2006/07 & 2007/08 & 2008/09 \\
\hline & & & & & Proj. & Proj. \\
\hline Revenue (excluding grants) & 11.9 & 12.2 & 12.5 & 12.7 & 13.3 & 13.9 \\
\hline Domestic primary expenditure & 15.4 & 14.4 & 14.1 & 14.9 & 14.2 & 16.0 \\
\hline Current expenditures net of interest payments & 11.5 & 10.9 & 10.9 & 10.5 & 10.5 & 9.2 \\
\hline of which: wages and salaries & 4.9 & 4.8 & 4.8 & 4.7 & 4.6 & 4.0 \\
\hline other current & 6.6 & 6.1 & 6.1 & 5.8 & 5.9 & 5.1 \\
\hline Domestically-financed development expenditures & 3.1 & 3.0 & 2.9 & 3.4 & 3.0 & 5.4 \\
\hline Of which: additional energy crisis spending & 0.0 & 0.0 & 0.0 & 1.0 & 0.4 & 0.6 \\
\hline Net lending and investment & 0.2 & 0.0 & -0.2 & 0.2 & -0.6 & -0.1 \\
\hline Of which: Bujagali bridge loan & 0.0 & 0.0 & 0.0 & 0.6 & -0.5 & 0.0 \\
\hline Other spending & 0.6 & 0.5 & 0.5 & 0.7 & 1.2 & 1.5 \\
\hline Of which: repayment of arrears & 0.3 & 0.4 & 0.5 & 0.7 & 1.2 & 1.2 \\
\hline Domestic primary balance & -3.5 & -2.2 & -1.6 & -2.1 & -0.9 & -2.1 \\
\hline Adjusted domestic primary balance ' & -3.2 & -1.8 & -1.1 & 0.2 & 0.1 & -0.3 \\
\hline
\end{tabular}

${ }^{1}$ Adjusted to exclude additional energy crisis spending, Bujagali-related net lending, and repayment of arrears.

b. Domestic borrowing of more than one percent of GDP will be required in 2008/09 (see Table 2). This, however, will reverse only a fraction of the primary domestic adjustment of close to $3 \frac{1}{2}$ percentage points of GDP achieved in recent years (text table).

\section{There are two pressure points that will need careful monitoring going forward.}

On the revenue side, the programmed $1 / 2$ of GDP increase in tax revenues in 2008/09 is fairly ambitious, with the effects of ongoing improvements in revenue administration likely to be offset to some extent by slower growth in import-related taxes. ${ }^{4}$ The authorities are confident that the revenue objective can be attained, but administrative and policy measures beyond those currently being considered (MEFP, \6) may be necessary during the course of the year. Second, with the fiscal stance shifting, the pressure on the shilling to appreciate could become even more acute, should large foreign exchange inflows persist.

11. Fiscal consolidation is expected to resume in $\mathbf{2 0 0 9 / 1 0}$ on the assumption that increasing revenue would promote a reduction in the deficit, excluding grants, albeit from a higher level (Table 5). The cumulative rise in public debt of some four percent of GDP over the next three years would still leave net public debt at a low level-about 20 percent of GDP - by 2011/12. Staff will review the medium-term fiscal outlook in more detail in the context of the upcoming Article IV consultation.

\footnotetext{
${ }^{4}$ Taxes on fuel, for example, are specific and do not benefit from the global oil price surge.
} 


\section{B. Monetary Policy and Financial Sector}

12. The tasks of sterilizing the sizeable foreign exchange inflows and fighting the imported inflation dominate the monetary policy agenda, although the higher import bill from oil and capital goods is likely to considerably reduce the overall balance of payments surplus:

- $\quad$ There was broad agreement that rapid economic growth has played a minimal role in the pick-up in inflation and that the first-round effect of higher food and oil prices on local prices should be accommodated, on the assumption that they will have a permanent impact on the overall price level. Accordingly the base money target for end-June 2008 has been revised slightly upwards. To avoid second-round effects, the base money path through end-June 2009 is based on a conservative core inflation projection of five percent.

- With the burden of sterilizing foreign exchange inflows through open market operations falling predominantly on interest rates and the budget, the BOU is reviewing its sterilization mix. However, policy makers remain mindful of the potentially adverse effects that intensified appreciation of the shilling against the U.S. dollar could have on exporters.

13. The authorities attach great importance to further developing Uganda's financial sector. After a comprehensive review process, four new banks have been allowed entry, boosting competition in the banking sector. Credit to the private sector is expanding rapidly, and the quality of the banks' portfolios appears to remain sound (Table 8). To address the sector's medium-term challenges, the BOU is preparing the financial market development plan (MEFP, $\mid 13)$.

\section{Other Issues}

\section{With the construction of the Bujagali dam on track, the authorities have} identified the Karuma hydropower project as the next least-cost power generation option. The Bujagali project has been implemented as a Public-Private Partnership (PPP) initiative, with US\$115 of the US\$400 million non-concessional borrowing room in the program being utilized. The authorities are now exploring financing modalities for the Karuma project, including use of the resources accumulated in the energy fund and/or arrangements similar to the Bujagali project. In view of the macroeconomic significance of the project, the authorities - in line with the priorities set out in the debt strategy — also do not rule out borrowing on nonconcessional terms. The program accordingly includes borrowing room of up to US $\$ 400$ million or about three percent of GDP. The last joint Bank-Fund Debt Sustainability Analysis (Country Report 08/4) showed that such borrowing would not materially impact external debt sustainability. 
15. Both under-budgeting and non-observance of public financial management rules have contributed to further accumulation of domestic arrears. New CCS arrears accumulated in 2006/07 amounted to 0.3 percent of GDP. Concerned about the implications for effective expenditure control, the government is planning a series of measures to reinforce its efforts to eliminate such arrears (MEFP, $₫ 8)$.

\section{Limited availability of long-term credit is seen as a major constraint on private} sector development and industrialization. Accordingly, the government plans to devote about 0.2 percent of GDP annually to schemes intended to improve access to such credit by small and medium-size enterprises (MEFP, $\mid 5$ ). Staff argued that, while the budgetary cost of the initiatives in the pipeline are manageable, a more concerted push in this direction would take away funds for infrastructure - which has been identified as the dominant constraint on industrialization.

\section{Program Monitoring}

17. For monitoring performance under the program, quantitative assessment criteria, quantitative indicative targets, and structural assessment criteria and benchmarks have been set (MEFP, Tables 1 and 2):

a. The end-June 2008 quantitative assessment criteria on net international reserves and net domestic assets of the BOU have been modified to reflect strong foreign exchange inflows. The assessment criterion on net credit to government has been modified to reflect new, higher, external financing assumptions for 2007/08. The assessment criterion on nonconcessional borrowing has been modified to allow financing for the Karuma hydropower project on nonconcessional terms. The indicative target on poverty-related spending has been modified to exclude outcomes not under direct control of the Ministry of Finance (TMU, $\mid 10)$.

b. It is now clear that comprehensive data on arrears accumulation (and verification) is only possible after the end of each fiscal year, with the introduction of IFMS having proved insufficient to allow for timely reporting Pending the authorities' review of the IFMS, the definition of domestic arrears is being modified to exclude arrears accumulated during the current fiscal year and the end-June 2008 indicative target has been revised accordingly. (TMU, ๆ8).

c. An additional structural benchmark — on the regulatory framework for pension funds - has been added. 


\section{Staff Appraisal}

18. Staff supports the authorities' request for waivers for the nonobservance of the assessment criteria and modification of quantitative assessment criteria, and recommends completion of the third review. Uganda's private sector-driven economy, assisted by the disciplined conduct of fiscal and monetary policies, has shown remarkable resilience in the face of shocks. The economic outlook remains positive. The nonobserved structural assessment criteria largely stem from pragmatic adjustments rather than significant deviations from program goals.

19. Given Uganda's infrastructure bottlenecks, the increase in public investment is appropriate. While the macroeconomic risks of higher deficit-financed expenditure appear manageable, this does not preclude the need for careful project appraisal and timely implementation. Care should be taken to ensure that the budgeted compression of current expenditures in 2008/09 does not give rise to new arrears or disruptions to key public services.

20. Strong efforts to address the long-standing problem of domestic arrears are required. The clearing of old arrears is proceeding apace, yet the accumulation of new arrears has not been arrested. Staff welcomes the authorities' continued focus on this issue and the proposed new measures. But if these measures are to reverse the increasing trend in arrears, much more political commitment will be needed, particularly in light of the proposed squeeze on current spending.

21. The BOU will need to stand ready to tighten monetary policy if risks of inflationary expectations arise. In dealing with high world food and fuel prices, the BOU is employing an appropriate combination of short-term accommodation and forward-looking tightening in setting the targets for base money. In implementing monetary policy, allowing for a higher degree of exchange rate flexibility may be required to ease the budgetary cost of sterilization.

22. Uncertain repercussions from the global economic slowdown represent the main risk to the program. Slower growth in the industrial countries could dampen export prices, although this could be offset by lower prices of imports. In addition, continued strife in the North could erase recent economic gains in the region.

23. Uganda appears to be in a relatively comfortable position to withstand the food and oil price shocks due to a strong economy, sufficient locally-grown food supply, and substantial foreign exchange reserves. At the same time, additional funding may be needed to alleviate adverse effects on some segments of the population (drought affected regions) and internally displaced persons in the North. 
Table 1. Uganda: Selected Economic and Financial Indicators, 2005/06-2008/09 ${ }^{1}$

\begin{tabular}{|c|c|c|c|c|c|}
\hline & \multirow{2}{*}{$\begin{array}{r}2005 / 06 \\
\text { Act. }\end{array}$} & \multirow{2}{*}{$\begin{array}{r}2006 / 07 \\
\text { Act. } \\
\end{array}$} & \multicolumn{2}{|c|}{$2007 / 08$} & \multirow{2}{*}{$\begin{array}{r}2008 / 09 \\
\text { Proj. }\end{array}$} \\
\hline & & & Prog. & Proj. & \\
\hline \multicolumn{6}{|l|}{ GDP and prices (percent change) } \\
\hline Real GDP & 10.8 & 7.9 & 7.1 & 9.8 & 8.1 \\
\hline Headline inflation (end of period) & 7.2 & 4.4 & 5.1 & 10.2 & 4.5 \\
\hline Core inflation (end of period) & 5.2 & 7.3 & 4.8 & 11.3 & 5.0 \\
\hline \multicolumn{6}{|l|}{ External sector (percent change) } \\
\hline Terms of trade (deterioration -) & 18.2 & 7.7 & -4.0 & 3.4 & -6.6 \\
\hline Real effective exchange rate (depreciation -) & -1.5 & 2.7 & $\ldots$ & $\ldots$ & $\ldots$ \\
\hline \multicolumn{6}{|l|}{ Money and credit (percent change) } \\
\hline Broad money (M2) & 18.9 & 16.7 & 16.6 & 20.3 & 14.5 \\
\hline Domestic credit & 7.2 & -6.4 & 14.3 & 7.2 & 15.4 \\
\hline Credit to the central government ${ }^{2}$ & -4.8 & -17.2 & 0.8 & -7.6 & 4.0 \\
\hline Private sector credit & 28.3 & 22.9 & 28.7 & 31.4 & 22.2 \\
\hline \multicolumn{6}{|l|}{ Savings and investment (percent of GDP) } \\
\hline Domestic investment & 21.2 & 22.3 & 27.6 & 24.1 & 25.2 \\
\hline Public & 4.4 & 4.8 & 7.0 & 4.9 & 5.9 \\
\hline Private & 16.8 & 17.5 & 20.6 & 19.2 & 19.2 \\
\hline National savings (excluding grants) & 13.0 & 14.9 & 15.0 & 15.4 & 13.8 \\
\hline Public & -0.9 & -0.8 & -0.8 & -2.8 & -2.1 \\
\hline Private & 13.9 & 15.8 & 15.7 & 18.2 & 16.0 \\
\hline \multicolumn{6}{|l|}{ External sector (percent of GDP) } \\
\hline Current account balance (including grants) & -3.5 & -2.8 & -8.0 & -3.6 & -6.7 \\
\hline Net donor inflows & 7.2 & 7.6 & 8.1 & 7.7 & 6.9 \\
\hline Current account balance (excluding grants) & -8.1 & -7.4 & -12.6 & -8.8 & -11.3 \\
\hline External debt (including Fund) & 43.0 & 11.1 & 15.9 & 14.3 & 16.6 \\
\hline External debt-service ratio ${ }^{3,4}$ & 7.3 & 1.2 & 5.0 & 2.6 & 2.1 \\
\hline \multicolumn{6}{|l|}{ Government budget and debt (percent of GDP) } \\
\hline Revenue & 12.5 & 12.7 & 14.1 & 13.3 & 13.9 \\
\hline Grants & 5.4 & 4.6 & 4.3 & 5.3 & 4.4 \\
\hline Total expenditure and net lending & -17.8 & -18.3 & -21.8 & -21.0 & -22.0 \\
\hline Overall balance (including grants) & 0.1 & -1.0 & -3.4 & -2.4 & -3.7 \\
\hline Overall balance (excluding grants) & -5.3 & -5.6 & -7.7 & -7.7 & -8.1 \\
\hline Stock of domestic debt & 8.7 & 9.4 & 9.9 & 11.6 & 10.2 \\
\hline \multicolumn{6}{|l|}{ Memorandum items: } \\
\hline Nominal GDP (U Sh billions) & 18,172 & 20,953 & 22,223 & 24,069 & 28,101 \\
\hline Average exchange rate (U Sh per US\$) & 1,825 & 1,778 & $\ldots$ & $\ldots$ & $\ldots$ \\
\hline Treasury bill yield (percent) & 7.8 & 9.4 & $\ldots$ & $\ldots$ & $\ldots$ \\
\hline Overall balance of payments (US\$ millions) & 198 & 704 & 86 & 569 & 21 \\
\hline \multicolumn{6}{|l|}{ Gross foreign exchange reserves } \\
\hline (months of next year's imports of goods and services) & 4.8 & 5.8 & 5.2 & 6.0 & 5.6 \\
\hline
\end{tabular}

Sources: Ugandan authorities; and IMF staff estimates and projections.

1 Fiscal year begins in July.

${ }^{2}$ Percent of M3 at start of the period.

${ }^{3}$ Percent of exports of goods and nonfactor services.

${ }^{4}$ Including Fund obligations; reflects actual debt service paid, including debt relief. 
Table 2. Uganda: Fiscal Operations of the Central Government, 2005/06-2008/09 ${ }^{1}$

\begin{tabular}{|c|c|c|c|c|c|}
\hline & \multirow{2}{*}{$\begin{array}{r}\text { 2005/06 } \\
\text { Actual }\end{array}$} & \multirow{2}{*}{$\begin{array}{r}\text { 2006/07 } \\
\text { Actual }\end{array}$} & \multicolumn{2}{|c|}{$2007 / 08$} & \multirow{2}{*}{$\begin{array}{r}\text { 2008/09 } \\
\text { Proj }\end{array}$} \\
\hline & & & Prog. & Proj. & \\
\hline & \multicolumn{2}{|c|}{ (U Sh Billions) } & & & \\
\hline Total revenue and grants & 3,241 & 3,624 & 4,085 & 4,464 & 5,140 \\
\hline Revenue & 2,267 & 2,667 & 3,135 & 3,197 & 3,906 \\
\hline Tax & 2,142 & 2,524 & 2,965 & 3,074 & 3,759 \\
\hline International trade taxes & 227 & 261 & 297 & 309 & 369 \\
\hline Income taxes & 653 & 782 & 924 & 910 & 1,186 \\
\hline Excises & 567 & 653 & 802 & 804 & 914 \\
\hline Value-added tax & 696 & 829 & 931 & 1,051 & 1,283 \\
\hline Additional revenue effort & 0 & 0 & 12 & 0 & 6 \\
\hline Nontax & 125 & 144 & 170 & 123 & 147 \\
\hline Grants & 974 & 957 & 949 & 1,267 & 1,234 \\
\hline Budget support & 738 & 776 & 481 & 520 & 503 \\
\hline Of which: MDRI (IMF) & 232 & $\ldots$ & $\ldots$ & $\ldots$ & $\ldots$ \\
\hline Project grants & 236 & 181 & 468 & 747 & 731 \\
\hline Expenditures and net lending & 3,228 & 3,844 & 4,842 & 5,051 & 6,176 \\
\hline Current expenditures & 2,233 & 2,442 & 2,733 & 2,842 & 2,934 \\
\hline Wages and salaries & 867 & 987 & 1,112 & 1,115 & 1,138 \\
\hline Interest payments & 249 & 236 & 297 & 309 & 356 \\
\hline Other current & 1,117 & 1,220 & 1,324 & 1,418 & 1,440 \\
\hline Development expenditures & 935 & 1,205 & 1,875 & 2,054 & 2,852 \\
\hline Donor-supported projects & 416 & 491 & 1,170 & 1,330 & 1,331 \\
\hline Domestic-financed & 519 & 714 & 704 & 724 & 1,521 \\
\hline Of which: add'I energy crisis spending & $\ldots$ & 207 & 92 & 92 & 160 \\
\hline Net lending and investment & -29 & 46 & -165 & -138 & -40 \\
\hline Other spending $^{2}$ & 91 & 151 & 400 & 293 & 430 \\
\hline \multicolumn{6}{|l|}{ Overall balance } \\
\hline Including grants & 13 & -220 & -757 & -587 & $-1,036$ \\
\hline Excluding grants & -962 & $-1,177$ & $-1,707$ & $-1,854$ & $-2,270$ \\
\hline Excl. grants and add'l energy spending & $\ldots$ & -970 & $-1,615$ & $-1,762$ & $-2,110$ \\
\hline Financing & -87 & 174 & 757 & 587 & 1,036 \\
\hline External financing (net) & 118 & 379 & 696 & 849 & 649 \\
\hline Disbursement & 317 & 520 & 893 & 978 & 778 \\
\hline Budget support & 76 & 249 & 191 & 395 & 178 \\
\hline Project loans & 241 & 271 & 702 & 583 & 600 \\
\hline Amortization (-) & -149 & -104 & -132 & -92 & -78 \\
\hline Exceptional financing & -50 & -38 & -66 & -36 & -53 \\
\hline Domestic financing (net) & -204 & -205 & 62 & -263 & 388 \\
\hline Bank financing & -135 & -562 & 31 & -294 & 188 \\
\hline Of which: MDRI-related government deposits & $\ldots$ & 56 & 44 & 44 & 55 \\
\hline Nonbank financing & -69 & 357 & 31 & 31 & 200 \\
\hline Errors and omissions/financing gap & 74 & 46 & 0 & 0 & 0 \\
\hline \multicolumn{6}{|l|}{ Memorandum items: } \\
\hline MDRI spending & $\ldots$ & 56 & 44 & 44 & 55 \\
\hline MDRI debt relief from IDA & $\ldots$ & 4,541 & $\ldots$ & $\ldots$ & $\ldots$ \\
\hline MDRI debt relief from the AfDF & $\ldots$ & 934 & $\ldots$ & $\ldots$ & $\ldots$ \\
\hline
\end{tabular}


Table 2. Uganda: Fiscal Operations of the Central Government, 2005/06-2008/09 ${ }^{1}$ (concluded)

\begin{tabular}{|c|c|c|c|c|c|}
\hline & $2005 / 06$ & $2006 / 07$ & 200 & & $2008 / 09$ \\
\hline & Actual & Actual & Prog. & Proj. & Proj. \\
\hline & (Percen & ff GDP) & & & \\
\hline Total revenue and grants & 17.8 & 17.3 & 18.4 & 18.5 & 18.3 \\
\hline Revenue & 12.5 & 12.7 & 14.1 & 13.3 & 13.9 \\
\hline Tax & 11.8 & 12.0 & 13.3 & 12.8 & 13.4 \\
\hline International trade taxes & 1.2 & 1.2 & 1.3 & 1.3 & 1.3 \\
\hline Income taxes & 3.6 & 3.7 & 4.2 & 3.8 & 4.2 \\
\hline Excises & 3.1 & 3.1 & 3.6 & 3.3 & 3.3 \\
\hline Value-added tax & 3.8 & 4.0 & 4.2 & 4.4 & 4.6 \\
\hline Additional revenue effort & 0.0 & 0.0 & 0.1 & 0.0 & 0.0 \\
\hline Nontax & 0.7 & 0.7 & 0.8 & 0.5 & 0.5 \\
\hline Grants & 5.4 & 4.6 & 4.3 & 5.3 & 4.4 \\
\hline Budget support & 4.1 & 3.7 & 2.2 & 2.2 & 1.8 \\
\hline Of which: MDRI (IMF) & 1.3 & $\ldots$ & $\ldots$ & $\ldots$ & \\
\hline Project grants & 1.3 & 0.9 & 2.1 & 3.1 & 2.6 \\
\hline Expenditures and net lending & 17.8 & 18.3 & 21.8 & 21.0 & 22.0 \\
\hline Current expenditures & 12.3 & 11.7 & 12.3 & 11.8 & 10.4 \\
\hline Wages and salaries & 4.8 & 4.7 & 5.0 & 4.6 & 4.0 \\
\hline Interest payments & 1.4 & 1.1 & 1.3 & 1.3 & 1.3 \\
\hline Other current & 6.1 & 5.8 & 6.0 & 5.9 & 5.1 \\
\hline Development expenditures & 5.1 & 5.8 & 8.4 & 8.5 & 10.2 \\
\hline Donor-supported projects & 2.3 & 2.3 & 5.3 & 5.5 & 4.7 \\
\hline Domestic-financed & 2.9 & 3.4 & 3.2 & 3.0 & 5.4 \\
\hline Of which: add'l energy crisis spending & $\ldots$ & 1.0 & 0.4 & 0.4 & 0.6 \\
\hline Net lending and investment & -0.2 & 0.2 & -0.7 & -0.6 & -0.1 \\
\hline Other spending ${ }^{<}$ & 0.5 & 0.7 & 1.8 & 1.2 & 1.5 \\
\hline Overall balance & & & & & \\
\hline Including grants & 0.1 & -1.0 & -3.4 & -2.4 & -3.7 \\
\hline Excluding grants & -5.3 & -5.6 & -7.7 & -7.7 & -8.1 \\
\hline Excl. grants and add'I energy spending & $\ldots$ & -4.6 & -7.3 & -7.3 & -7.5 \\
\hline Financing & -0.5 & 0.8 & 3.4 & 2.4 & 3.7 \\
\hline External financing (net) & 0.6 & 1.8 & 3.1 & 3.5 & 2.3 \\
\hline Disbursement & 1.7 & 2.5 & 4.0 & 4.1 & 2.8 \\
\hline Budget support & 0.4 & 1.2 & 0.9 & 1.6 & 0.6 \\
\hline Project loans & 1.3 & 1.3 & 3.2 & 2.4 & 2.1 \\
\hline Amortization (-) & -0.8 & -0.5 & -0.6 & -0.4 & -0.3 \\
\hline Exceptional financing & -0.3 & -0.2 & -0.3 & -0.2 & -0.2 \\
\hline Domestic financing (net) & -1.1 & -1.0 & 0.3 & -1.1 & 1.4 \\
\hline Bank financing & -0.7 & -2.7 & 0.1 & -1.2 & 0.7 \\
\hline Nonbank financing & -0.4 & 1.7 & 0.1 & 0.1 & 0.7 \\
\hline Errors and omissions/financing gap & 0.4 & 0.2 & 0.0 & 0.0 & 0.0 \\
\hline Memorandum item: & & & & & \\
\hline Adjusted domestic primary balance ${ }^{3}$ & -1.1 & 0.2 & 0.0 & 0.1 & -0.3 \\
\hline MDRI spending & $\ldots$ & 0.3 & 0.2 & 0.2 & 0.2 \\
\hline MDRI debt relief from IDA & $\ldots$ & 21.7 & $\ldots$ & $\ldots$ & $\ldots$ \\
\hline MDRI debt relief from the AfDF & $\ldots$ & 4.5 & $\ldots$ & $\ldots$ & \\
\hline
\end{tabular}

Sources: Ugandan authorities; and IMF staff estimates and projections.

${ }^{1}$ Fiscal year begins in July.

2 Includes arrears.

${ }^{3}$ Adjusted to exclude additional energy crisis spending, Bujagali-related net lending, and repayment of arrears. 
Table 3a. Uganda: Monetary Accounts, 2005/06-2008/09 ${ }^{1}$

(U Sh billions; end of period, unless otherwise indicated)

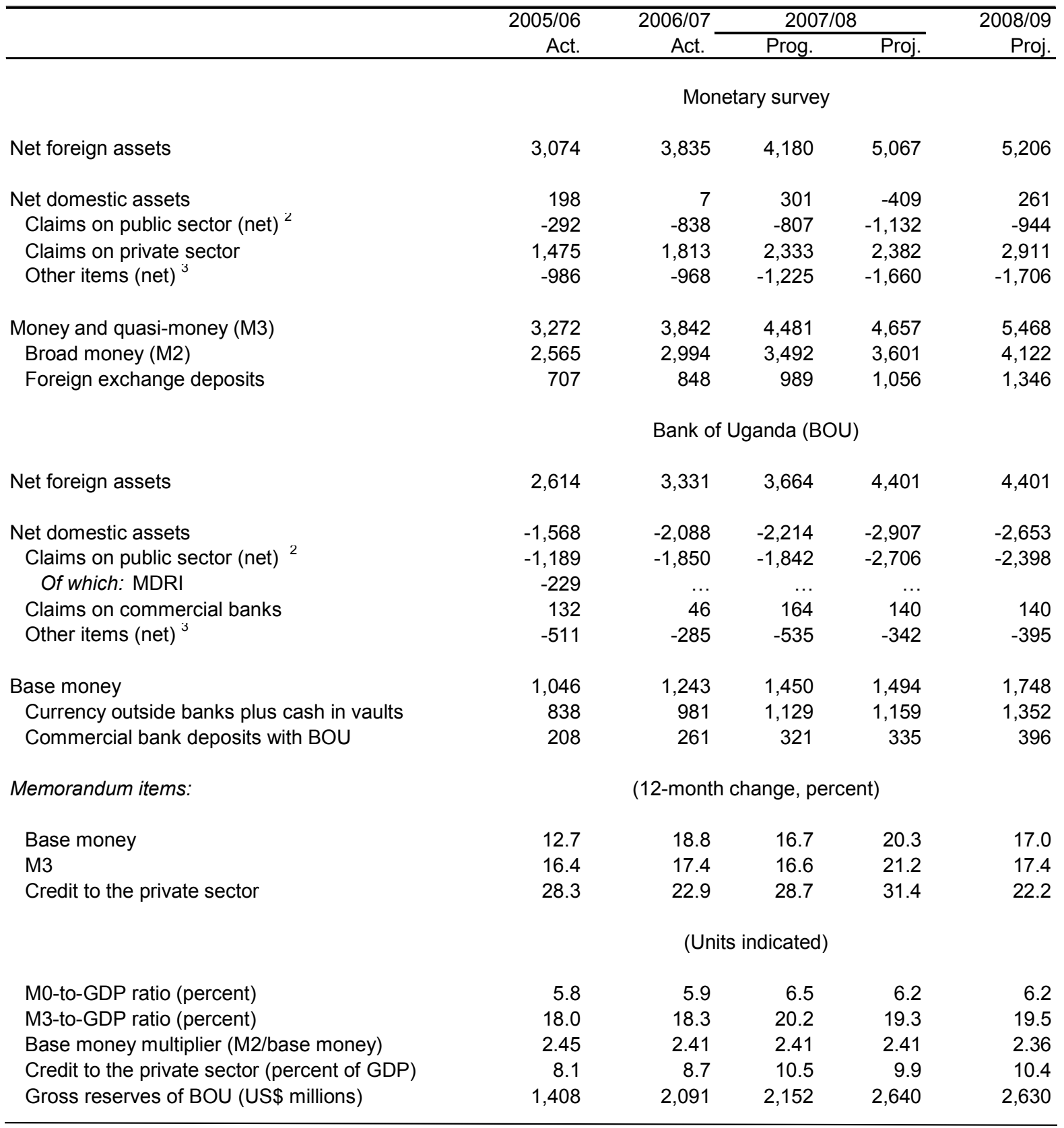

Sources: Ugandan authorities; and IMF staff estimates and projections.

${ }^{1}$ Fiscal year begins in July.

${ }^{2}$ The public sector includes the central government, the public enterprises, and the local government.

${ }^{3}$ Including valuation and the BOU's claims on private sector. 
Table 3b. Uganda: Quarterly Monetary Accounts, 2006/07-2008/09 ${ }^{1}$

( $U$ Sh billions; end of period, unless otherwise indicated)

\begin{tabular}{|c|c|c|c|c|c|c|c|c|c|}
\hline & \multirow{2}{*}{$\frac{2006 / 07}{\text { June }}$} & \multicolumn{4}{|c|}{$2007 / 08$} & \multicolumn{4}{|c|}{$2008 / 09$} \\
\hline & & Sep. & Dec. & Mar. & June $^{4}$ & Sep. $^{4}$ & Dec. $^{4}$ & Mar. $^{4}$ & June $^{4}$ \\
\hline & \multicolumn{9}{|c|}{ Monetary survey } \\
\hline Net foreign assets & 3,835 & 4,615 & 4,940 & 5,158 & 5,067 & 5,152 & 5,203 & 5,193 & 5,206 \\
\hline Net domestic assets & 7 & -429 & -517 & -369 & -409 & -196 & -143 & 400 & 261 \\
\hline Claims on public sector (net) ${ }^{2}$ & -838 & $-1,005$ & $-1,187$ & $-1,232$ & $-1,132$ & $-1,098$ & $-1,155$ & -858 & -944 \\
\hline Claims on private sector & 1,813 & 2,038 & 2,220 & 2,588 & 2,382 & 2,629 & 2,784 & 2,929 & 2,911 \\
\hline Other items (net) ${ }^{3}$ & -968 & $-1,462$ & $-1,550$ & $-1,725$ & $-1,660$ & $-1,727$ & $-1,772$ & $-1,671$ & $-1,706$ \\
\hline Money and quasi-money (M3) & 3,842 & 4,186 & 4,423 & 4,789 & 4,657 & 4,956 & 5,060 & 5,594 & 5,468 \\
\hline Broad money (M2) & 2,994 & 3,208 & 3,388 & 3,674 & 3,601 & 3,726 & 3,824 & 4,113 & 4,122 \\
\hline \multirow[t]{2}{*}{ Foreign exchange deposits } & 848 & 978 & 1,034 & 1,114 & 1,056 & 1,231 & 1,237 & 1,481 & 1,346 \\
\hline & \multicolumn{9}{|c|}{ Bank of Uganda (BOU) } \\
\hline Net foreign assets & 3,331 & 4,161 & 4,346 & 4,499 & 4,401 & 4,452 & 4,468 & 4,423 & 4,401 \\
\hline Net domestic assets & $-2,088$ & $-2,742$ & $-2,802$ & $-2,944$ & $-2,907$ & $-2,810$ & $-2,740$ & $-2,691$ & $-2,653$ \\
\hline $\begin{array}{l}\text { Claims on public sector (net) } \\
\text { Of which: MDRI }\end{array}$ & $-1,850$ & $-2,136$ & $-2,441$ & $-2,637$ & $-2,706$ & $-2,598$ & $-2,517$ & $-2,456$ & $-2,398$ \\
\hline Claims on commercial banks & 46 & 74 & 165 & 206 & 140 & 140 & 140 & 140 & 140 \\
\hline Other items (net) ${ }^{3}$ & -285 & -679 & -526 & -513 & -342 & -353 & -364 & -375 & -395 \\
\hline Base money & 1,243 & 1,420 & 1,544 & 1,554 & 1,494 & 1,641 & 1,727 & 1,732 & 1,748 \\
\hline Currency outside banks plus cash in vaults & 981 & 1,051 & 1,200 & 1,195 & 1,159 & 1,232 & 1,413 & 1,331 & 1,352 \\
\hline Commercial bank deposits with BOU & 261 & 368 & 344 & 359 & 335 & 410 & 315 & 401 & 396 \\
\hline Memorandum items: & \multicolumn{9}{|c|}{ (12-month change, percent) } \\
\hline Base money & 18.8 & 22.4 & 25.8 & 26.3 & 20.3 & 15.6 & 11.9 & 11.4 & 17.0 \\
\hline M3 & 17.4 & 22.0 & 22.0 & 23.6 & 21.2 & 18.4 & 14.4 & 16.8 & 17.4 \\
\hline Credit to the private sector & 22.9 & 29.4 & 29.3 & 48.8 & 31.4 & 29.0 & 25.4 & 13.2 & 22.2 \\
\hline & \multicolumn{9}{|c|}{ (Units indicated) } \\
\hline Base money multiplier (M2/base money) & 2.41 & 2.26 & 2.20 & 2.36 & 2.41 & 2.27 & 2.21 & 2.37 & 2.36 \\
\hline Gross reserves of BOU (US\$ millions) & 2,091 & 2,361 & 2,559 & 2,606 & 2,640 & 2,667 & 2,675 & 2,645 & 2,630 \\
\hline
\end{tabular}

Sources: Ugandan authorities; and IMF staff estimates and projections.

${ }^{7}$ Fiscal year begins in July.

${ }^{2}$ The public sector includes the central government, the public enterprises, and the local government.

${ }^{3}$ Including valuation and the BOU's claims on private sector.

${ }^{4}$ Projections 
Table 4. Uganda: Balance of Payments, 2005/06-2008/09 ${ }^{1}$

(US\$ millions)

\begin{tabular}{|c|c|c|c|c|c|}
\hline & $2005 / 06$ & $2006 / 07$ & 200 & & $2008 / 09$ \\
\hline & Act. & Act. & Prog. & Proj. & Proj. \\
\hline Current account & -350 & -331 & $-1,030$ & -505 & $-1,179$ \\
\hline Percent of GDP & -4.1 & -3.2 & -7.3 & -3.6 & -6.7 \\
\hline Trade balance & -926 & -993 & $-1,820$ & $-1,439$ & $-2,099$ \\
\hline Percent of GDP & -10.9 & -9.7 & -12.9 & -10.2 & -12.0 \\
\hline Exports, f.o.b. & 1,042 & 1,521 & 1,436 & 1,719 & 1,872 \\
\hline Of which: coffee & 173 & 229 & 239 & 254 & 255 \\
\hline Imports, f.o.b. & $-1,969$ & $-2,514$ & $-3,256$ & $-3,159$ & $-3,971$ \\
\hline Of which: oil & -290 & -403 & -464 & -605 & -812 \\
\hline Services (net) & -340 & -537 & -560 & -663 & -690 \\
\hline Income (net) & -238 & -224 & -358 & -335 & -392 \\
\hline Of which: interest on public debt & -35 & -22 & -36 & -31 & -23 \\
\hline Transfers & 1,155 & 1,423 & 1,708 & 1,931 & 2,001 \\
\hline Private transfers & 695 & 884 & 1,124 & 1,200 & 1,194 \\
\hline Of which: nongovernmental organizations & 430 & 524 & 663 & 867 & 768 \\
\hline Official transfers & 460 & 540 & 585 & 732 & 807 \\
\hline Of which: budget support & 219 & 359 & 231 & 259 & 262 \\
\hline project support & 129 & 102 & 270 & 389 & 456 \\
\hline HIPC grants & 58 & 77 & 47 & 46 & 52 \\
\hline Capital and financial account & 557 & 1,002 & 1,116 & 970 & 1,201 \\
\hline Capital account & 127 & 3,428 & 0 & 0 & 0 \\
\hline Of which: MDRI debt relief & 127 & 3,428 & 0 & 0 & 0 \\
\hline Financial account & 430 & $-2,426$ & 1,116 & 970 & 1,201 \\
\hline Foreign direct and portfolio investment & 368 & 518 & 570 & 558 & 638 \\
\hline Other investment & 61 & $-2,944$ & 546 & 411 & 562 \\
\hline Medium- and long-term & 118 & $-3,091$ & 722 & 661 & 766 \\
\hline Of which: & & & & & \\
\hline Public sector (net) & 84 & $-3,145$ & 514 & 405 & 437 \\
\hline Disbursements & 166 & 342 & 590 & 460 & 486 \\
\hline Project support & 132 & 153 & 405 & 274 & 375 \\
\hline Budget support & 34 & 264 & 110 & 110 & 111 \\
\hline Amortization due & -82 & $-3,487$ & -76 & -54 & -49 \\
\hline Private sector (net) & 34 & 54 & 208 & 255 & 329 \\
\hline Short-term & -57 & 148 & -175 & -249 & -204 \\
\hline Errors and omissions & -11 & 32 & 0 & 104 & 0 \\
\hline Overall balance & 198 & 704 & 86 & 569 & 21 \\
\hline Financing & -198 & -704 & -86 & -569 & -21 \\
\hline Central bank net reserves (increase $=-$ ) & -163 & -682 & -50 & -549 & 10 \\
\hline Of which: gross reserve change & -24 & -682 & -50 & -549 & 10 \\
\hline Memorandum items: & & & & & \\
\hline Coffee export volume (millions of 60-kg. bags) & 2.1 & 2.5 & 2.6 & 2.3 & 2.4 \\
\hline Coffee price (U.S. cents per kg.) & 137 & 150 & 152 & 180 & 176 \\
\hline Net donor support & 611 & 778 & 1,037 & 1,091 & 1,203 \\
\hline Of which: budget support & 253 & 623 & 341 & 370 & 373 \\
\hline project support & 261 & 254 & 674 & 663 & 831 \\
\hline total HIPC debt relief & 78 & 49 & 38 & 49 & 48 \\
\hline
\end{tabular}

Sources: Ugandan authorities; and IMF staff estimates and projections.

${ }^{1}$ Fiscal year begins on July 1 . 
Table 5. Uganda: Medium-Term Macroeconomic Framework, 2005/06-2009/10 ${ }^{1}$

\begin{tabular}{|c|c|c|c|c|c|}
\hline & $\begin{array}{r}2005 / 06 \\
\text { Act. }\end{array}$ & $\begin{array}{r}2006 / 07 \\
\text { Act. }\end{array}$ & $\begin{array}{r}2007 / 08 \\
\text { Proj. }\end{array}$ & $\begin{array}{r}2008 / 09 \\
\text { Proj. }\end{array}$ & $\begin{array}{r}2009 / 10 \\
\text { Proj. }\end{array}$ \\
\hline \multicolumn{6}{|l|}{ National income and prices } \\
\hline Real GDP (percent change) & 10.8 & 7.9 & 9.8 & 8.1 & 8.0 \\
\hline Per capita GDP (2004/05 US\$) & 370 & 387 & 412 & 432 & 452 \\
\hline Underlying average inflation (percent change) & 5.2 & 7.8 & 8.0 & 8.0 & 5.0 \\
\hline Gross domestic investment (percent of GDP) & 21.2 & 22.3 & 24.1 & 25.2 & 25.9 \\
\hline \multicolumn{6}{|l|}{$\begin{array}{l}\text { External sector } \\
\quad \text { (percent of GDP, unless otherwise indicated) }\end{array}$} \\
\hline Current account balance (including grants) & -3.5 & -2.8 & -3.6 & -6.7 & -6.9 \\
\hline Net donor inflows & 7.2 & 7.6 & 7.7 & 6.9 & 5.9 \\
\hline Current account balance (excluding grants) & -8.1 & -7.4 & -8.8 & -11.3 & -11.0 \\
\hline NPV of debt-to-exports ratio (percent) & -- & -- & 48.8 & 58.6 & 61.4 \\
\hline \multicolumn{6}{|l|}{ Government budget and debt (percent of GDP) } \\
\hline Revenue & 12.5 & 12.7 & 13.3 & 13.9 & 14.4 \\
\hline Grants & 5.4 & 4.6 & 5.3 & 4.4 & 3.4 \\
\hline Total expenditure and net lending & 17.8 & 18.3 & 21.0 & 22.0 & 21.7 \\
\hline Overall balance (including grants) & 0.1 & -1.0 & -2.4 & -3.7 & -3.9 \\
\hline Overall balance (excluding grants) & -5.3 & -5.6 & -7.7 & -8.1 & -7.3 \\
\hline Stock of domestic debt & 8.7 & 9.4 & 11.6 & 10.2 & 8.3 \\
\hline Credit to the private sector (percent of GDP) & 8.1 & 8.7 & 9.9 & 10.4 & 11.0 \\
\hline \multirow{2}{*}{\multicolumn{6}{|c|}{ Gross foreign exchange reserves }} \\
\hline & 4.8 & 5.8 & 6.0 & 5.6 & 5.5 \\
\hline
\end{tabular}

Sources: Ugandan authorities; and IMF staff estimates and projections.

1 Fiscal year begins in July. 


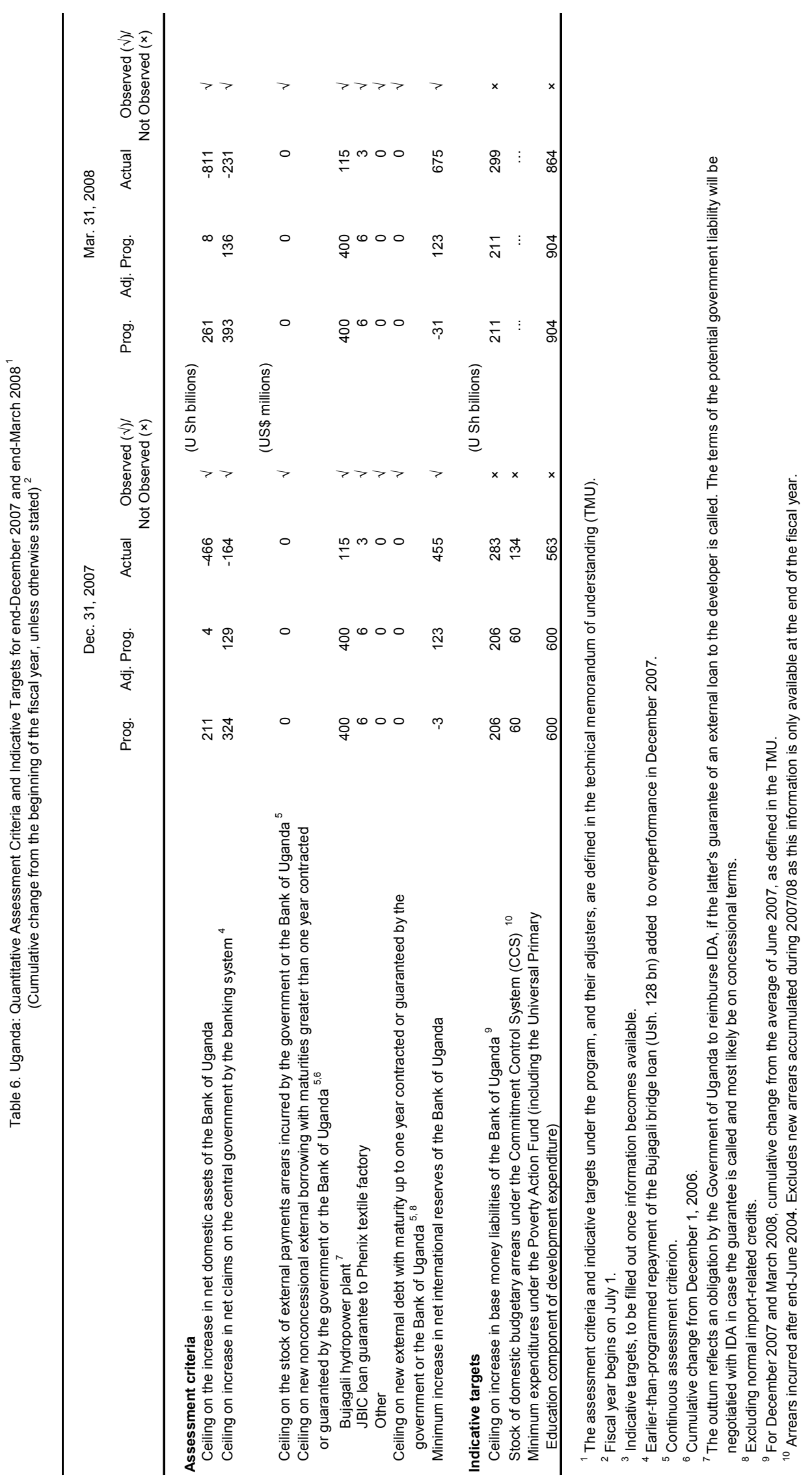


Table 7. Uganda: Structural Assessment Criteria and Benchmarks ${ }^{1}$

Date of

Implementation
Implementation Status

\section{Structural Assessment Criteria}

1. Finalize and publish the Government of Uganda Debt Strategy.

2. Submit to cabinet a policy paper outlining the establishment of a new regulatory framework for financial institutions not under the statutory supervision of the BOU. These financial institutions will include private and public pension funds, and the Uganda Development Bank.

3. Implement pilot Integrated Personnel and Payroll System in three Commissions (Public Service Commission, Health Service Commission, Education Service Commission), Ministries (Ministry of Finance, Ministry of Health, Ministry of Public Service, Ministry of Education), and Local Governments (Lira and Jinja Districts).

4. For $2008 / 09$ budget, allocate U Sh 300 billion for payments of verified group (A) domestic arrears, with priority given to pension arrears. ${ }^{2}$

5. Issue a tender to select the provider for the national identity card system.

\section{End-December Observed 2007}

End-June 2008

Not observed. The framework for supervision of pension funds has been submitted to Cabinet. The insurance industry is already being regulated by the insurance commission. An appropriate entity to regulate SACCOs will be found. The framework for the UDB will not be established due to the UDB's relatively small size.

End-May 2008

Not observed. Implementation date shifted to January 2009 due to delays in procurement process. $^{3}$

End-June 2008

Not observed. The resources will be allocated for payments of all verified arrears. $^{3}$

End-June 2008

Not observed. Due to cost considerations, introduction of the national ID system to be postponed. Priority will be given to enhancing the existing databases. 
Table 7. Uganda: Structural Assessment Criteria and Benchmarks (concluded) ${ }^{1}$

\section{Policy Measure}

Date of Implementation Implementation Status

\section{Structural Benchmarks}

6. The BOU, in consultation with other members of the Monetary Affairs Committee of the EAC, will finalize a comprehensive draft End-May 2008 Observed financial market development strategy.

7. The Ministry of Finance, in conjunction with the Ministry of Foreign Affairs, will submit to Cabinet the complete list of

End-April 2008 Observed international subscriptions and the related payment obligations.

8. Update national energy (Oil) policy to include macroeconomic policy options.

End-June 2008 Observed

\footnotetext{
${ }^{1}$ Assessment criteria also apply on a continuous basis to the standard provisions on the exchange and trade issues that apply to programs supported by the Fund's financial resources.

${ }^{2}$ Group (A) arrears comprise the stock of pre-CCS, non-CCS, and CCS arrears incurred before end-June 2004.

${ }^{3}$ See Table 2 in the MEFP.
} 
Table 8. Uganda: Selected Banking Sector Information, June 2004-December 2007

\begin{tabular}{|c|c|c|c|c|c|c|c|c|}
\hline & \multicolumn{2}{|c|}{2004} & \multicolumn{2}{|c|}{2005} & \multicolumn{2}{|c|}{2006} & \multicolumn{2}{|c|}{2007} \\
\hline & June & Dec. & June & Dec. & June & Dec. & June & Dec. \\
\hline & \multicolumn{8}{|c|}{ (U Sh billions) } \\
\hline Adjusted assets & 3,184 & 3,294 & 3,526 & 3,659 & 3,841 & 4,422 & 4,629 & 5,546 \\
\hline Liquid assets & 1,279 & 1,538 & 1,374 & 1,558 & 1,179 & 1,500 & 1,460 & 1,688 \\
\hline Tier I & 276 & 313 & 311 & 348 & 379 & 444 & 480 & 526 \\
\hline Tier II & 32 & 30 & 26 & 32 & 36 & 40 & 35 & 50 \\
\hline Total capital & 309 & 343 & 337 & 380 & 416 & 484 & 515 & 575 \\
\hline Risk-weighted assets (RWA) & 1,624 & 1,674 & 1,881 & 2,076 & 2,271 & 2,686 & 2,563 & 2,957 \\
\hline Nonperforming assets (NPA) & 14 & 21 & 33 & 29 & 41 & 50 & 60 & 89 \\
\hline Loans and advances & 884 & 977 & 1,114 & 1,258 & 1,450 & 1,703 & 1,768 & 2,174 \\
\hline Foreign exchange advances & 218 & 267 & 327 & 384 & 450 & 463 & 465 & 650 \\
\hline Foreign exchange depostis & 806 & 817 & 838 & 786 & 815 & 949 & 937 & 1,150 \\
\hline \multirow[t]{2}{*}{ Deposits } & 2,396 & 2,438 & 2,549 & 2,595 & 2,741 & 2,961 & 3,145 & 3,613 \\
\hline & \multicolumn{8}{|c|}{ (Percent) } \\
\hline Return on average assets & 5.0 & 4.3 & 3.4 & 3.6 & 3.5 & 3.4 & 3.9 & 3.9 \\
\hline Core capital/ RWA & 17.0 & 18.7 & 16.5 & 16.8 & 16.7 & 16.5 & 18.7 & 17.8 \\
\hline Total capital/ RWA & 19.0 & 20.5 & 17.9 & 18.3 & 18.3 & 18.0 & 20.1 & 19.5 \\
\hline NPAs/total advances & 1.6 & 2.2 & 2.9 & 2.3 & 2.8 & 2.9 & 3.4 & 4.1 \\
\hline Loan loss provision/ NPA & 139.6 & 97.8 & 78.0 & 103.8 & 93.7 & 74.4 & 69.3 & 71.8 \\
\hline Liquid assets/ total deposits & 53.4 & 63.1 & 53.9 & 60.0 & 43.0 & 50.7 & 46.4 & 46.7 \\
\hline Advances/ deposits & 36.9 & 37.4 & 43.7 & 48.5 & 52.9 & 57.5 & 56.2 & 60.2 \\
\hline Foreign exchange exposure/ core capital ${ }^{1}$ & 4.7 & -2.0 & -2.7 & -2.7 & -8.7 & -7.4 & -2.5 & -8.4 \\
\hline Foreign exchange advances/ foreign exchange deposits & 27.0 & 32.6 & 39.1 & 48.8 & 55.2 & 48.8 & 49.6 & 56.6 \\
\hline
\end{tabular}

Source: Ugandan authorities.

${ }^{1}$ Foreign exchange exposure is calculated using the short-hand method. 
Table 9. Uganda: Millennium Development Goals

\begin{tabular}{|c|c|c|c|c|}
\hline & 1990 & 1995 & 2001 & 2005 \\
\hline Eradicate extreme poverty and hunger. & \multicolumn{4}{|c|}{2015 target = halve $1990 \$ 1$ a day poverty and malnutrition rates } \\
\hline Percentage share of income or consumption held by poorest 20 percent & $\ldots$ & $\ldots$ & 5.9 & $\ldots$ \\
\hline Prevalence of child malnutrition (percent of children under 5) & 23.0 & 25.5 & 22.8 & $\ldots$ \\
\hline Population below minimum level of dietary energy consumption (percent) & 23.0 & 25.0 & 19.0 & $\cdots$ \\
\hline Achieve universal primary education. & \multicolumn{4}{|c|}{2015 target $=$ net enrollment to 100} \\
\hline Net primary enrollment ratio (percent of relevant age group) & $\ldots$ & 87.3 & $\ldots$ & $98.4^{1}$ \\
\hline Youth literacy rate (percent of ages 15-24) & 70.1 & 74.7 & 79.4 & 77.0 \\
\hline Promote gender equality. & \multicolumn{4}{|c|}{2015 target $=$ education ratio to 100} \\
\hline Ratio of girls to boys in primary and secondary education (percent) & 76.8 & 81.0 & 95.0 & 96.0 \\
\hline Ratio of young literate females to males (percent of ages 15-24) & 75.8 & 80.4 & 85.0 & 86.0 \\
\hline Share of women employed in the nonagricultural sector (percent) & 43.2 & $\ldots$ & $\ldots$ & $\ldots$ \\
\hline Proportion of seats held by women in national parliament (percent) & 12.0 & 17.0 & 18.0 & 24.0 \\
\hline Reduce child mortality. & \multicolumn{4}{|c|}{2015 target $=$ reduce 1990 under -5 mortality by two-thirds } \\
\hline Under-5 mortality rate (per 1,000 ) & 160.0 & 156.0 & 145.0 & 136.0 \\
\hline Infant mortality rate (per 1,000 live births) & 93.0 & 92.0 & 85.0 & 79.0 \\
\hline Immunization, measles (percent of children under 12 months) & 52.0 & 57.0 & 61.0 & 86.0 \\
\hline Improve maternal health. & \multicolumn{4}{|c|}{2015 target $=$ reduce 1990 maternal mortality by three-fourths } \\
\hline Maternal mortality ratio (modeled estimate, per 100,000 live births) & $\ldots$ & $\ldots$ & 880.0 & $\ldots$ \\
\hline Births attended by skilled health staff (percent of total) & 38.3 & 37.8 & 39.0 & $\ldots$ \\
\hline Combat HIVIAIDS, malaria, and other diseases. & \multicolumn{4}{|c|}{2015 target $=$ halt, and begin to reverse, AIDS, etc. } \\
\hline Prevalence of HIV, female (percent of ages 15-24) & $\ldots$ & $\ldots$ & 4.6 & 5.0 \\
\hline Contraceptive prevalence rate (percent of women ages 15-49) & 4.9 & 14.8 & 22.8 & $\ldots$ \\
\hline Number of children orphaned by HIVIAIDS (thousands) & $\ldots$ & $\ldots$ & 880.0 & $\ldots$ \\
\hline Incidence of tuberculosis (per 100,000 people) & 161.1 & $\ldots$ & 324.0 & 369.0 \\
\hline Tuberculosis cases detected under DOTS (percent) ${ }^{2}$ & $\ldots$ & $\ldots$ & 44.4 & 45.0 \\
\hline Ensure environmental sustainability. & \multicolumn{4}{|c|}{2015 target $=$ various } \\
\hline Forest area (percent of total land area) & 25.9 & $\ldots$ & 21.3 & 18.0 \\
\hline Nationally protected areas (percent of total land area) & $\ldots$ & 9.7 & 9.7 & 32.6 \\
\hline CO2 emissions (metric tons per capita) & 0.0 & 0.0 & 0.1 & 0.1 \\
\hline Access to an improved water source (percent of population) & 45.0 & $\ldots$ & 52.0 & 60.0 \\
\hline Access to improved sanitation (percent of population) & $\cdots$ & $\cdots$ & 79.0 & 43.0 \\
\hline Develop the Global Partnership for Development. & \multicolumn{4}{|c|}{2015 target $=$ various } \\
\hline Fixed line and mobile telephones (per 1,000 people) & 1.6 & 2.0 & 13.5 & 56.0 \\
\hline Personal computers (per 1,000 people) & $\ldots$ & 0.5 & 2.8 & 9.0 \\
\hline \multicolumn{5}{|l|}{ General indicators } \\
\hline Population (millions) & 17.8 & 20.9 & 25.1 & 28.8 \\
\hline Adult literacy rate (percent of people ages 15 and over) & 56.1 & $\ldots$ & $\ldots$ & 67.0 \\
\hline Total fertility rate (births per woman) & 7.2 & 6.9 & $\ldots$ & 7.1 \\
\hline Life expectancy at birth (years) & 45.7 & 43.1 & $\ldots$ & 50.0 \\
\hline
\end{tabular}

Source: World Development Indicators database, April 2007.

1 Data for 2004.

2 Percentage of estimated new infectious tuberculosis cases detected under the directly observed treatment, short-course case detection and treatment strategy. 
Mr. Dominique Strauss Kahn

Managing Director

International Monetary Fund

Washington, D.C. 20431

Dear Mr. Strauss Kahn:

On behalf of the Government of Uganda, I would like to inform you of the progress we have made under our economic program backed by the Fund's Policy Support Instrument (PSI) and transmit the attached Memorandum of Economic and Financial policies (MEFP), which sets out the objectives and policies that the Government intends to pursue in the short and medium term. The policies outlined in the MEFP are drawn from the Government's initiatives to promote employment and rapid economic growth in the context of a stable macroeconomic environment.

Despite a less favorable global economic environment and the disruptions caused by the recent turmoil in Kenya, the economy continues to grow at high rates, partly taking advantage of the improved trading environment in the region. Fiscal developments in 2007/08 are also set to be in line with the our objectives.

Most assessment criteria for the third review under the PSI were observed. We did, however, introduce changes to some of the structural measures in our program - (i) establishment of new framework for financial institutions not supervised by the BOU, (ii) implementation of the Integrated Personnel and Payroll System pilot, (iii) issuing a tender to select the provider for the national ID card system, and (iv) allocation of budgetary resources to arrears clearance - in light of new information and reassessment of costs and priorities. Taking into account the progress achieved in the implementation of the program and the clarifications provided below, the Government of Uganda requests waivers for nonobservance of the relevant assessment criteria.

The Government of Uganda believes that the policies set forth in the MEFP are adequate to achieve the objectives of our PSI-supported program. Given our interest in macroeconomic stability, we stand ready to take additional measures as may be necessary to achieve needed objectives. Our PSI proposes assessment criteria for the performance target dates of end-June and end-December 2008 for the fourth and fifth reviews, which are expected to be completed by end- November 2008 and end-April 2009. We stand ready to work with the Fund and other development partners in the implementation of our program and will consult in advance should revisions be contemplated to the policies contained in the PSI. 
The Government of Uganda authorizes the publication and distribution of this letter, its attachments, and all reports prepared by Fund staff regarding the current PSI review.

Sincerely yours,

$/ \mathrm{s} /$

Dr. Ezra Suruma

Minister of Finance, Planning, and Economic Development 


\title{
APPENDIX I-ATTACHMENT I \\ Uganda: Memorandum of Economic And Financial Policies
}

\begin{abstract}
UPDATE
1. The Government of Uganda remains committed to sustained macroeconomic stability, economic growth, and poverty reduction. This updated Memorandum of Economic and Financial Policies (MEFP) summarizes the Government's strategy to achieve these goals as set out in the Poverty Eradication Action Plan (PEAP). The Government and the International Monetary Fund (IMF) are cooperating on the economic program through a three-year Policy Support Instrument (PSI). This MEFP describes performance under the program through May 2008, specific policies and targets through the first half of 2008/09, and medium-term objectives.
\end{abstract}

\section{Performance Under the PSI}

2. All assessment criteria have been met. Fiscal performance in the first half of 2007/08 was well within the program limits and, accordingly, the ceiling on net claims on government by the banking system was observed. The ceiling on net domestic assets of the Bank of Uganda (BOU) and the floor on the stock of net international reserves of the BOU were also comfortably observed. Base money exceeded only marginally the allowed 5 percent band with respect to the indicative target. The indicative target for poverty related spending performed at 94 percent of programmed amounts, marginally below the 95 percent target. The debt strategy was published in December 2007; and U Sh 300 billion has been allocated for arrears clearance in 2008/09 budget. The other structural measures are being modified as outlined below.

\section{Objectives and Policies Looking Forward}

3. The central objective of our economic policies is to raise economic growth to achieve rapid and sustained poverty reduction. Without this higher rate of growth, job creation will not keep pace with the half million new entrants into the labor market each year. Supported by macroeconomic stability and sound public finances, our policies will continue to focus on addressing the key constraints to higher private investment. An improved security situation in northern Uganda and peace and stability in the broader east and central Africa region are also expected to give the economy a fillip. Recent macroeconomic developments have been heavily influenced by strong balance of payments performance, including capital inflows, with attendant appreciation pressures on the Uganda shilling. Our near-term financial policies are being calibrated with a view to addressing these pressures. 


\section{A. Fiscal Policy}

4. The outlook for the remainder of the 2007/08 fiscal year ending in June is in line with the Medium-Term Fiscal Framework (MTFF) and program objectives set out in the previous MEFP.

- $\quad$ The turmoil in Kenya has not had a significant impact on fiscal aggregates. Better than expected tax collection in the first half of 2007/08, has fully offset the negative impact of the crisis on customs revenues in January. And when calm returned, the flow of goods through Kenya reverted back to normal levels. For the full year, we expect revenues to be some U Sh 70 billion better than targeted.

- Despite significant unexpected pressures, total spending has been kept within the budget envelope. In the first half of the fiscal year, the successfully concluded Commonwealth Heads of Government Meeting required greater resources than originally allocated in the budget. Responses to emergencies, including floods and an Ebola outbreak, required additional spending. The decision to invest in a new government aircraft for use by the President also entailed additional outlays. Cumulatively, spending on these items amounted to 0.3 percent of GDP, and was financed from a combination of cuts in non-priority spending and higher-thanexpected revenues. The Presidential Affairs Committee of Parliament has been briefed about the acquisition of the new aircraft, and the old one will be sold consistent with national regulations to ensure transparency and highest return to the government. Overall, we expect to remain within the program's fiscal targets for 2007/08.

5. The 2008/09 budget will significantly increase agriculture and infrastructure spending to address bottlenecks to economic growth and poverty reduction. While ongoing measures to strengthen tax collection and reduction in current spending will help to create most of the required fiscal space, we will also increase domestic borrowing modestly. However, given the sizeable fiscal consolidation of recent years, fiscal sustainability will not be put at risk. The areas that will receive significantly more funding are:

- $\quad$ Agriculture. With a view to increasing farm productivity, we are working on improving the value chain including research and extension services to farmers. We are also reviewing policies to provide to farmers better access to inputs, financing and marketing services.

- Infrastructure. The government will sustain the increase in allocation towards infrastructure initiated in the last two budgets, with a focus on energy and transportation. 
- Energy. With the help of a US\$75 million bridge loan by the GOU, construction of the hydroelectric dam and power plant at Bujagali has been underway for close to a year. Financing for the project was secured by the project sponsor in December, and the bridge loan has been repaid. In the event, only US\$115 of the borrowing room under the non-concessional borrowing limit of US $\$ 400$ million has been used. We are now turning our attention to the Karuma hydro project, which has been identified as the next least cost option and will be financed by a mix of public and private resources (the former including the moneys from the government's energy fund). We are working with the project developers on financing modalities. In the event concessional financing cannot be secured, we intend to use non-concessional borrowing for the project and are requesting a US $\$ 400$ million limit on nonconcessional borrowing in the PSI-supported program.

- $\quad$ Roads. With respect to transportation projects, we are actively looking at ways to facilitate the flow of goods in and out of the country by using turnkey projects and improving implementation through the formation of the National Roads Authority and creation of Road Fund. Specifically, the government over the next three years plans to spend an additional US\$200 million per year to upgrade the key transport corridors linking our country to South Sudan, Kenya, and Rwanda, consistent with national procurement regulations. The appropriation under the line item "transport corridor project" will be ringfenced and only used for the construction of these key road corridors, with any additional funds needed beyond US $\$ 200$ million being financed from reallocations within the budget envelope.

- Railway. We are reviewing the concession agreement with Rift Valley Railway to ensure its implementation.

- $\quad$ Human resource development. The 2008/09 budget will also increase investment in human capital development, with a particular emphasis on (i) expanding Universal Primary and Secondary Education access and (ii) better basic health service delivery with a view to reducing high maternal and infant mortality rates.

- $\quad$ Private sector development. The problem of limited value addition is a result of coordination and market failures and we plan to address this through the following initiatives:

- The construction of industrial parks where concentrated infrastructure services can be better provided;

- Gradual recapitalization of the Uganda Development Bank (UDB) to ensure that it can provide long-term financing - the absence of which we have 
identified as a major impediment to the growth of small and medium scale enterprises. Government intends to issue an Industrialization Bond in 2008/09 of U Sh 20 billion to be managed by UDB, with the possibility of more resources being allocated to the bank in the medium term providing the resources are used effectively. UDB will extend term loans on a basis that ensures full cost recovery, and the performance of the loans will be judged against internationally accepted best practices for development banks. Should the Bank's non-performing loan (NPL) ratio exceed 10 percent, additional resources will not be provided to the Bank until it brings the ratio back below this level. Annual independent audits will be required on the quality of UDB's risk assessment before additional resources are provided to recapitalize UDB.

- Government intends to introduce a credit guarantee scheme, to underwrite risk to encourage commercial banks to lend to the agricultural sector. Under the scheme, the government will provide a guarantee for up to 50 percent of the credit risk associated with loans by commercial banks for certain sectors. By using the banks superior credit assessment capacity, the scheme ensures that resources will be directed to the most viable projects. The 2008/09 budget will allocate U Sh 20 billion for this scheme.

6. The Government remains committed to increasing tax collection in the medium term. Revenue collections in 2007/08 were strong reflecting administrative improvements in the Uganda Revenue Authority following the reforms that have been undertaken. But there is still more scope to mobilize more tax resources through further improvements in tax administration. Accordingly we plan to:

- $\quad$ Produce a tax procedure code that will help individuals and businesses better understand their tax obligations and improve tax compliance.

- Introduce amendments to the Income Tax Act for petroleum taxation.

- Update the excise tax law and take steps to address the tax revenue loss as a result of smuggling.

- $\quad$ Take measures to increase the efficiency of the tax tribunal by appointing members with adequate qualifications by end-June 2008.

- $\quad$ Consider the merits of introducing an electronic tax registry, to improve the VAT collection.

7. Parliament has recently approved tax incentives for qualified investors to take effect starting July 1, 2008. Revenue losses are expected to be very modest (not exceeding 0.1 percent of GDP), with the incentives only being granted to companies exporting more than 80 percent of their production. Coupled with the other initiatives noted above, this 
implies that the budget is altogether allocating some U Sh 60 billion (1/4 percent of GDP) for private sector development. Going forward, these initiatives will be the modalities through which the government will provide sector-specific support. Other government resources will be directed towards the other priorities noted above.

8. The Government remains committed to reducing arrears, notwithstanding the increase in new arrears in 2006/07 to U Sh 100 billion from some U Sh 60 billion in the previous two fiscal years. Moreover, two thirds of the arrears incurred in 2006/07 was in areas covered by the commitment control system. This suggests that the system is not working as effectively as envisaged. The problem for some spending categories (such as utilities) is partly due to insufficient budgetary allocations. But failure by line ministries and agencies to observe public financial management rules also plays a role. To address the challenge, we are undertaking the following initiatives:

- $\quad$ For 2008/09, we will ensure sufficient allocation of resources and minimize cuts once spending limits have been allocated.

- Given the weakness evident in the control mechanisms under IFMS, we are launching a comprehensive audit of the system which will inter alia review the effectiveness of the system, including with respect to access, compliance, and the system's integrity. This review is expected to be completed by end-September, 2008.

- $\quad$ Starting in July 2008 to coincide with the new fiscal year, to control the accumulation of CCS arrears, expenditures committed outside the IFMS system for both IFMS and non-IFMS votes will not be recognized by the Accountant General as arrears and therefore will not be programmed for payment in future budgets by MoFPED. Efforts will be made to ensure that clearance of category B arrears takes the first call on resources available on a quarterly basis.

- $\quad$ Consistent with repeated notifications by Cabinet and MOFPED to spending agencies that arrears would be cleared out of future budgetary allocations, starting with the 2008/09 budget, up to 15 percent of quarterly cash releases to spending agencies will be withheld and used to clear verified arrears, until the cumulative CCS arrears incurred by the relevant spending agency since the start of the 2004/05 fiscal year has been cleared.

- The Ministry of Public Service will implement the new Integrated Personnel and Payroll System in three Commissions (Public Service Commission, Health Service Commission, Education Service Commission), four Ministries (Ministry of Finance, Ministry of Health, Ministry of Public Service, Ministry of Education), and two local governments (Lira and Jinja Districts) by January 2009. This should improve payroll and pension records and ensure accurate payroll and pension figures. The 
implementation deadline is being extended from May 2008 due to delays in the procurement process.

- $\quad$ To address the arrears to international organizations, the Ministry of Finance, together with the Ministry of Foreign Affairs, have submitted to cabinet the complete list of international subscriptions and the related payment obligations. The individual memberships will be reviewed and sufficient budgetary provisions ensured.

\section{B. Monetary and Financial Sector Policies}

9. We remain committed to keeping annual average core inflation below five percent guided by our reserve money target. In 2007/08, inflation is likely to trend higher than this on account of strong increases in international fuel, food, and commodity prices and effects of the turmoil in Kenya. Given higher economic growth and an increase in money demand, the Bank of Uganda (BOU) will increase the targeted stock of base money for June 2008 by an additional 3 percent. For 2008/09, base money growth will target core inflation of 5 percent.

10. The BOU will continue to manage liquidity using an appropriate instrument mix. Subject to the conditions in the foreign exchange market, BOU will continue to use sales of foreign exchange to sterilize donor inflows. In view of the persistent inflows, we will keep under review the instrument mix with a view to avoiding undue upward pressure on interest rates.

11. The BOU is pursuing the tools needed for a future inflation targeting framework. In June 2007, UBOS rolled out a new measure of core inflation that would more accurately capture inflationary trends and is expected to roll out a quarterly GDP index by October 2008. 


\section{Other Structural Reforms}

12. The government has decided on specialized regulatory agencies for the non-bank financial entities. There is already an insurance commission which regulates the insurance industry. For pensions, we have decided to establish a specific regulatory agency for existing (including NSSF) and new entities. Draft legislation establishing the new body will be submitted to parliament by end-December, 2008. An appropriate entity will be found to supervise the non-deposit taking financial service providers, such as leasing companies, Savings and Credit Cooperatives (SACCOs), and other microfinance institutions. The Government recognizes the need to supervise the Uganda Development Bank (UDB), but at this stage does not wish to establish a dedicated regulatory body to supervise a single relatively small institution (its balance sheet is approximately US\$30 million). At this stage, the UDB's activities are being monitored closely by its newly reconstituted and independent board. As the UDB's portfolio expands, however, the Government will find an appropriate regulatory solution.

13. As part of the Monetary Affairs Committee of the EAC, the BOU has drafted a fiveyear financial market development plan and has received comments from the key stakeholders that will soon be incorporated. The plan represents an approach to addressing the current limitations in Uganda's financial markets by identifying gaps and constraints. It also sets a foundation for implementation of proposed recommendations by assigning different roles to various stakeholders.

14. The introduction of a national identity card system is now expected to take somewhat longer than previously envisaged, partly due to limited resources to cover its high cost. In the interim, the government is taking a number of steps to enhance existing databases (for passports, driving licenses, tax identification numbers, etc) including by supplementing them with biometric information. In the financial sector, the challenge of minimizing identity fraud-related loses will now be addressed through the issuance of financial cards, which will include biometric information of the individual bank customers, that will be issued by the Credit Reference Bureau (CRB).

15. The government has reached an agreement on the principles guiding the operation of the energy fund and will issue appropriate regulations. The Ministry of Finance will have the authority to decide on using the energy fund. Like the general government account, the energy fund will be subject to the review by the Auditor General.

16. Commercial oil production is expected to start soon. We recognize the challenges associated with the increased revenues, including their potentially negative impact on macroeconomic stability, fiscal sustainability, and development of the non-oil economy. To ensure that the oil wealth is used wisely, Government has finalized the national energy policy and is updating it to include macroeconomic policy options. Government will ensure that prudent policies, transparency and accountability of operations are in place. 
Table 1. Uganda: Quantitative Assessment Criteria and Indicative Targets for June 2008 - June $2009^{1}$ (Cumulative change from the beginning of the fiscal year, unless otherwise stated) ${ }^{2}$

\begin{tabular}{|c|c|c|c|c|c|}
\hline & \multicolumn{2}{|c|}{$\begin{array}{c}\text { June } 30 \\
2008\end{array}$} & \multirow{2}{*}{$\begin{array}{c}\text { Sept. } 30 \\
2008^{3} \\
\text { Prog. }\end{array}$} & \multirow{2}{*}{$\begin{array}{c}\text { Dec. } 31 \\
2008 \\
\text { Prog. }\end{array}$} & \multirow{2}{*}{$\begin{array}{c}\text { June } 30 \\
2009^{3} \\
\text { Prog. }\end{array}$} \\
\hline & Prog. & Rev. prog. & & & \\
\hline Assessment criteria & \multicolumn{5}{|c|}{ (U Sh billions) } \\
\hline Ceiling on the increase in net domestic assets of the Bank of Uganda ${ }^{4}$ & 125 & -504 & 100 & 174 & 270 \\
\hline \multirow[t]{2}{*}{ Ceiling on the increase in net claims on the central government by the banking system ${ }^{4}$} & 31 & -308 & 34 & -23 & 188 \\
\hline & \multicolumn{5}{|c|}{ (US\$ millions) } \\
\hline \multirow{2}{*}{\multicolumn{6}{|c|}{$\begin{array}{l}\text { Ceiling on new nonconcessional external borrowing with maturities greater than one year contracted } \\
\text { or guaranteed by the government or the Bank of Uganda }{ }^{5,6}\end{array}$}} \\
\hline & & & & & \\
\hline Karuma hydropower plant & $\cdots$ & 400 & 400 & 400 & 400 \\
\hline JBIC loan guarantee to Phenix textile factory & 6 & 6 & 6 & 6 & 6 \\
\hline Other & 0 & 0 & 0 & 0 & 0 \\
\hline \multicolumn{6}{|l|}{ Ceiling on new external debt with maturity up to one year contracted or guaranteed by the } \\
\hline government or the Bank of Uganda ${ }^{5,7}$ & 0 & 0 & 0 & 0 & 0 \\
\hline Minimum increase in net international reserves of the Bank of Uganda & 50 & 449 & 28 & 35 & -10 \\
\hline Indicative target & \multicolumn{5}{|c|}{ (U Sh billions ) } \\
\hline Ceiling on the increase in base money liabilities of the Bank of Uganda ${ }^{4}$ & 208 & 252 & 147 & 233 & 254 \\
\hline Stock of domestic budgetary arrears under the Commitment Control System (CCS) ${ }^{8}$ & 30 & 125 & $\ldots$ & $\ldots$ & $\ldots$ \\
\hline $\begin{array}{l}\text { Minimum expenditures under the Poverty Action Fund (including the Universal Primary } \\
\text { Education component of development expenditure) }\end{array}$ & 1,222 & 1,222 & 298 & 596 & 1,192 \\
\hline
\end{tabular}

${ }^{1}$ The assessment criteria and indicative targets under the program, and their adjusters, are defined in the TMU.

2 Fiscal year begins on July 1.

3 Indicative target.

${ }^{4}$ For June 2008, cumulative changes from the average of June 2007. For September and December 2008, and for June 2009, cumulative changes from average of June 2008, as defined in the TMU.

${ }^{5}$ Continuous performance criterion.

${ }^{6}$ Cumulative change from December 1, 2006.

${ }^{7}$ Excluding normal import-related credits.

${ }^{8}$ Arrears incurred after end-June 2004. Excludes new arrears accumulated during the current fiscal year. 
Table 2. Uganda: Structural Assessment Criterion and Benchmark ${ }^{1}$

\begin{tabular}{ccc}
\hline Policy Measure & $\begin{array}{c}\text { Date of } \\
\text { Implementation }\end{array}$ & $\begin{array}{c}\text { Implementation } \\
\text { Status }\end{array}$ \\
\hline
\end{tabular}

\section{Structural Assessment Criterion}

Implement pilot Integrated Personnel and Payroll System in three

Commissions (Public Service Commission, Health Service

End-January

2009

Commission, Education Service Commission), Ministries (Ministry of

Finance, Ministry of Health, Ministry of Public Service, Ministry of

Education), and Local Governments (Lira and Jinja Districts).

\section{Structural Benchmark}

Submit to Parliament draft regulatory framework for pension funds. End-December 2008

\footnotetext{
${ }^{1}$ Assessment criteria also apply on a continuous basis to the standard provisions on the exchange and trade issues that apply to programs supported by the Fund's financial resources.
} 


\section{APPENDIX I-ATTACHMENT II \\ Uganda: TECHNICAL MEMORANDUM OF UNDERSTANDING}

\section{A. Introduction}

1. This memorandum defines the targets described in the memorandum of economic and financial policies (MEFP) for the July 2006-June 2009 financial program supported by the IMF Policy Support Instrument (PSI), and sets forth the reporting requirements under the instrument.

\section{B. Net Foreign Assets (NFA) and Net Domestic Assets (NDA) of the Bank of Uganda (BOU)}

2. The net foreign assets of the BOU are defined as the monthly average (based on daily data) of foreign assets minus foreign liabilities, and include all foreign claims and liabilities of the central bank. The monthly average values of all foreign assets and liabilities will be converted into U.S. dollars at each test date using the average cross exchange rates for March 2008 for the various currencies and then converted into Uganda shillings using the average U.S. dollar-Uganda shilling exchange rate for March 2008.

\begin{tabular}{|l|l|}
\hline \multicolumn{2}{|c|}{ Program Exchange Rates (US\$ per currency unit, unless indicated otherwise) } \\
\hline Euro & 1.5500 \\
\hline British pound & 2.0033 \\
\hline Japanese Yen & 0.0099 \\
\hline Kenya shilling & 0.0153 \\
\hline Tanzania shilling & 0.0008 \\
\hline SDR & 1.6304 \\
\hline Uganda shilling (per US\$1) & $1,684.26$ \\
\hline
\end{tabular}

3. Net domestic assets (NDA) of the Bank of Uganda (BOU) are defined as the monthly average (based on daily data) of base money (defined below) less net foreign assets of the BOU (as defined in para. 2). Based on this definition, the NDA limits will be cumulative changes from the average of June 2007 to the average of June 2008, and cumulative changes from the average of June 2008 to the average of September and December 2008, and June 
2009. The respective cumulative changes are increases of U Sh -504 billion, U Sh 100 billion, U Sh 174 billion, and U Sh 270 billion.

\begin{tabular}{|c|c|c|c|c|}
\hline \multicolumn{5}{|l|}{ (In billions of shillings) } \\
\hline & June 2008 & Sept 2008 & Dec 2008 & June 2009 \\
\hline $\begin{array}{l}\text { Cumulative change in } \\
\text { base money }\end{array}$ & 252 & 147 & 233 & 254 \\
\hline $\begin{array}{l}\text { Cumulative change in } \\
\text { NFA }\end{array}$ & 756 & 47 & 59 & -16 \\
\hline $\begin{array}{l}\text { Cumulative change in } \\
\text { NDA }\end{array}$ & -504 & 100 & 174 & 270 \\
\hline
\end{tabular}

\section{B. Base Money}

4. Base money is defined as the sum of currency issued by Bank of Uganda (BOU) and the commercial banks' deposits in the BOU. The commercial bank deposits include the statutory required reserves and excess reserves held at the BOU and are net of the deposits of closed banks at the BOU and Development Finance Funds (DFF) contributed by commercial banks held at the BOU. The base money limits will be cumulative changes from the daily average of June 2007 to the daily average of June 2008, and cumulative changes from the daily average of June 2008 to the daily average of September and December 2008, and June 2009.

\section{Net Claims on the Central Government by the Banking System}

5. Net claims on the central government (NCG) by the banking system is defined as the difference between the outstanding amount of bank credits to the central government and the central government's deposits with the banking system, excluding deposits in administered accounts and project accounts with the banking system, including the central bank. Credits comprise bank loans and advances to the government and holdings of government securities and promissory notes. Central government's deposits with the banking system include the full amount of IMF MDRI. NCG will be calculated based on data from balance sheets of the monetary authority and commercial banks as per the monetary survey. The quarterly limits on the change in NCG by the banking system will be cumulative beginning end-June in the previous fiscal year. 


\section{Net International Reserves of the Bank of Uganda}

6. Net international reserves (NIR) of the BOU are defined for program monitoring purpose as reserve assets of the BOU net of short-term external liabilities of the BOU. Reserve assets are defined as external assets readily available to, and controlled by, the BOU and exclude pledged or otherwise encumbered external assets, including, but not limited to, assets used as collateral or guarantees for third-party liabilities. Short-term external liabilities are defined as liabilities to nonresidents, of original maturities less than one year, contracted by the BOU and include outstanding IMF purchases and loans.

7. For program-monitoring purposes, reserve assets and short-term liabilities at the end of each test period will be calculated in U.S. dollars by converting them from their original currency denomination at program exchange rates (as specified in para. 2).

\section{E. Ceiling on Domestic Budgetary Arrears of the Central Government}

8. The stock of domestic payment arrears under the Commitment Controls System (CCS) will be monitored on a quarterly basis. Domestic payments arrears under the CCS are defined as the sum of all bills that have been received by a central government spending unit or line ministry delivered prior to the beginning of the current fiscal year, and for which payment has not been made within 30 days under the recurrent expenditure budget (excluding court awards) or the development expenditure budget. For the purpose of program monitoring, the quarterly CCS reports, which will include arrears accumulated at IFMIS and non-IFMIS sites, prepared by the Internal Audit and Inspection Office will be used to monitor arrears. Arrears can be cleared in cash or through debt swaps.

9. The payments of pre-CCS, non-CCS, and CCS arrears accumulated up to end-June 2004 ("group A arrears") are covered by specific budget allocations for 2006/07 and 2007/08. The program ceiling on the stock of CCS arrears only covers accumulation of arrears after end-June 2004 ("group B arrears"). According to the verified report prepared by the Internal Audit and Inspection Office, this stock of arrears is estimated at U Sh 147 billion as of June 2007.

\section{F. Expenditures Under The Poverty Action Fund (PAF).}

10. The compliance with the indicative target on minimum expenditures under the PAF will be verified on the basis of releases (PAF resources made available to spending agencies).

\section{G. Adjusters}

11. The NDA and NIR targets are based on program assumptions regarding budget support, assistance provided under the Heavily Indebted Poor Countries (HIPC) Initiative and the Multilateral Debt Relief Initiative (MDRI), external debt-service payments, and 
automatic access by commercial banks to the BOU's rediscount and discount window facilities. The NCG target, in addition to being based on the aforementioned assumptions, is also based on assumptions regarding domestic nonbank financing of central government fiscal operations. In addition, the NDA target depends on the legal reserve requirements on deposits in commercial banks.

The Uganda shilling equivalent of budget support (grants and loans) plus HIPC Initiative assistance in the form of grants on a cumulative basis from July 1 of the fiscal year is presented under Schedule A. The ceilings on the cumulative increase in NDA and NCG will be adjusted downward (upward), and the floor on the cumulative increase in NIR of the BOU will be adjusted upward (downward) by the amount by which budget support, grants and loans, plus HIPC Initiative and MDRI assistance, exceeds (falls short of) the projected amounts.

\begin{tabular}{|l|c|c|c|c|}
\hline \multicolumn{5}{|c|}{$\begin{array}{c}\text { Schedule A: Budget Support Plus Total HIPC Initiative Assistance } \\
\text { (Cumulative billions of Uganda shillings, beginning July 1 of the fiscal year) }\end{array}$} \\
\hline Quarter & June 30, 2008 & $\begin{array}{c}\text { September 30, } \\
2008\end{array}$ & $\begin{array}{c}\text { December 31, } \\
2008\end{array}$ & June 30, 2009 \\
\hline $\begin{array}{l}\text { Budget support, including } \\
\text { HIPC Initiative grants }\end{array}$ & 915 & 238 & 384 & 681 \\
\hline
\end{tabular}

12. The ceiling on the increases in NDA and NCG will be adjusted downward (upward) and the floor on the increase in NIR will be adjusted upward (downward) by the amount by which debt service due ${ }^{5}$ plus payments of external debt arrears less deferred payments (exceptional financing) falls short of (exceeds) the projections presented in Schedule B. Deferred payments are defined to be (i) all debt service rescheduled under the HIPC Initiative; and (ii) payments falling due to all non-HIPC Initiative creditors that are not currently being serviced by the authorities (that is, gross new arrears being incurred).

\begin{tabular}{|l|c|c|c|c|}
\hline \multicolumn{5}{|c|}{$\begin{array}{c}\text { Schedule B: Debt Service Due, Before HIPC Initiative Assistance } \\
\text { (Cumulative billions of Uganda shillings, beginning July 1 of the fiscal year) }\end{array}$} \\
\hline Quarter & June 30, 2008 & September 30, 2008 & December 31, 2008 & June 30, 2009 \\
\hline $\begin{array}{l}\text { Debt service due before HIPC, } \\
\text { excluding exceptional } \\
\text { financing }\end{array}$ & 165 & 88 & 133 & 185 \\
\hline
\end{tabular}

\footnotetext{
${ }^{5}$ Debt service due is defined as pre-HIPC Initiative debt service due, but from 2003/04 onwards, this excludes HIPC Initiative debt rescheduling.
} 
13. The ceiling on the increase in NCG will be adjusted downward (upward) by any excess (shortfall) in nonbank financing ${ }^{6}$ less payment of domestic group A arrears relative to the programmed cumulative amounts presented in Schedule C. For the purpose of this adjuster, payment of domestic group A arrears cannot exceed the programmed amount by more than U Sh 45.0 billion.

\begin{tabular}{|l|c|c|c|c|}
\hline \multicolumn{5}{|c|}{$\begin{array}{c}\text { Schedule C: Nonbank Financing Minus Repayment of Domestic Arrears } \\
\text { (Cumulative billions of Uganda shillings, beginning July 1 of the fiscal year) }\end{array}$} \\
\hline Quarter & June 30, 2008 & $\begin{array}{c}\text { September 30, } \\
2008\end{array}$ & $\begin{array}{c}\text { December 31, } \\
2008\end{array}$ & June 30, 2009 \\
\hline $\begin{array}{l}\text { (A) Nonbank } \\
\text { financing }\end{array}$ & 31 & -22 & 14 & 200 \\
\hline $\begin{array}{l}\text { (B) Domestic arrears } \\
\text { repayment }\end{array}$ & 280 & 20 & 137 & 300 \\
\hline \begin{tabular}{l} 
(C) Total = (A) -(B) \\
\hline
\end{tabular} & -249 & -41 & -123 & -100 \\
\hline
\end{tabular}

14. The ceiling on NDA of the BOU for end-June will be adjusted upward by the daily average amount of commercial bank automatic access to the BOU discount window and rediscounting of government securities by commercial banks.

15. The ceiling on NDA of the BOU for every test date will be adjusted downward/upward to reflect decreases/increases in the legal reserve requirements on deposits in commercial banks. The adjuster will be calculated as the percent change in the reserve requirement multiplied by the actual amount of required reserves (Uganda shillings and foreign-currency denominated) at the end of the previous calendar month.

\section{H. External Borrowing Contracted or Guaranteed by the Central Government, Statutory Bodies, or the Bank of Uganda, and Arrears}

16. The assessment criterion on short-term debt refers to contracting or guaranteeing external debt with original maturity of one year or less by the government or the Bank of Uganda. Excluded from this assessment criterion are normal import-related credits. The definition of "debt" is set out in paragraph 18 .

\footnotetext{
${ }^{6}$ Comprising the check float and the change in government securities and government promissory notes held by the nonbank sector. The change in government securities held by the nonbank sector will be calculated from the data provided by the Central Depository System (CDS).
} 
17. The program includes a ceiling on new nonconcessional borrowing with maturities greater than one year contracted or guaranteed by the government, statutory bodies, or the BOU. ${ }^{7}$ Nonconcessional borrowing is defined as loans with a grant element of less than 35 percent, calculated using average commercial interest rates references (CIRRs) published by the Organization for Economic Cooperation and Development (OECD). In assessing the level of concessionality, the 10-year average CIRRs should be used to discount loans with maturities of at least 15 years, while the 6-month average CIRRs should be used for loans with shorter maturities. To both the 10-year and 6-month averages, the following margins for differing payment periods should be added: 0.75 percent for repayment periods of less than 15 years; 1 percent for $15-19$ years; 1.15 percent for 20-25 years; and 1.25 percent for 30 years or more. The ceiling on nonconcessional external borrowing or guarantees is to be observed on a continuous basis. The coverage of borrowing includes financial leases and other instruments giving rise to external liabilities, contingent or otherwise, on nonconcessional terms. Excluded from the limits are changes in indebtedness resulting from refinancing credits and rescheduling operations, and credits extended by the IMF. For the purposes of the program, arrangements to pay over time obligations arising from judicial awards to external creditors that have not complied with the HIPC Initiative do not constitute nonconcessional external borrowing. For the purposes of the program, the Bujagali project is defined as the hydroelectric dam and related equipment located at the dam site.

18. The definition of debt, for the purposes of the limit, is set out in point 9 of the Guidelines on Performance Criteria with Respect to External Debt (Executive Board's Decision No. 12274-(00/85), August 24, 2000). It not only applies to the debt as defined in Point 9 of the Executive Board decision, but also to commitments contracted or guaranteed for which value has not been received. The definition of debt set forth in No. 9 of the Guidelines on Performance Criteria with Respect to External Debt in Fund Arrangements reads as follows:

(a) For the purpose of this guideline, the term "debt" will be understood to mean a current, i.e., not contingent, liability, created under a contractual arrangement through the provision of value in the form of assets (including currency) or services, and which requires the obligor to make one or more payments in the form of assets (including currency) or services, at some future point(s) in time; these payments will discharge the principal and/or interest liabilities incurred under the contract. Debts can take a number of forms, the primary ones being as follows: (i) loans, i.e., advances of money to the obligor by the lender made on the basis of an undertaking that the obligor will repay the funds in the future (including deposits, bonds, debentures, commercial loans and buyers' credits) and temporary exchanges

\footnotetext{
${ }^{7}$ Contraction is defined as approval by a resolution of Parliament as required in Section 20(3) of the Public Finance and Accountability Act, 2003
} 
of assets that are equivalent to fully collateralized loans under which the obligor is required to repay the funds, and usually pay interest, by repurchasing the collateral from the buyer in the future (such as repurchase agreements and official swap arrangements); (ii) suppliers' credits, i.e., contracts where the supplier permits the obligor to defer payments until some time after the date on which the goods are delivered or services are provided; and (iii) leases, i.e., arrangements under which property is provided which the lessee has the right to use for one or more specified period(s) of time that are usually shorter than the total expected service life of the property, while the lesser retains the title to the property. For the purpose of the guideline, the debt is the present value (at the inception of the lease) of all lease payments expected to be made during the period of the agreement excluding those payments that cover the operation, repair, or maintenance of the property. (b) Under the definition of debt set out in point 9(a) above, arrears, penalties, and judicially awarded damages arising from the failure to make payment under a contractual obligation that constitutes debt. Failure to make payment on an obligation that is not considered debt under this definition (e.g., payment on delivery) will not give rise to debt.

19. The ceiling on the accumulation of new external payments arrears is zero. This limit, which is to be observed on a continuous basis, applies to the change in the stock of overdue payments on debt contracted or guaranteed by the government, the BOU, and statutory bodies ${ }^{8}$ from their level at end-June 2006. It comprises those external arrears reported by the Trade and External Debt Department of the BOU, the Macro Department of the Ministry of Finance that cannot be rescheduled because they were disbursed after the Paris Club cutoff date.

\section{Monitoring and Reporting Requirements}

20. The authorities will inform the IMF staff in writing at least ten business days (excluding legal holidays in Uganda or in the United States) prior to making any changes in economic and financial policies that could affect the outcome of the financial program. Such policies include but are not limited to customs and tax laws (including tax rates, exemptions, allowances, and thresholds), wage policy, and financial support to public and private enterprises. The authorities will similarly inform the IMF staff of any nonconcessional external debt contracted or guaranteed by the government, the BOU, or any statutory bodies, and any accumulation of new external payments arrears on the debt contracted or guaranteed

\footnotetext{
${ }^{8}$ This definition is consistent with the coverage of public sector borrowing defined by the Fund (includes the debt of the general government, monetary authorities, and entities that are public corporations which are subject to the control by government units, defined as the ability to determine general corporate policy or by at least 50 percent government ownership).
} 
by these entities. The authorities will furnish an official communication to the IMF describing program performance of quantitative and structural assessment criteria and benchmarks within 8 weeks of a test date. The authorities will on a regular basis submit information to IMF staff with the frequency and submission time lag as indicated in Table 1. The information should be mailed electronically to AFRUGA746@IMF.ORG . 
Table 1. Summary of Reporting Requirements

\begin{tabular}{|c|c|c|c|}
\hline $\begin{array}{l}\text { Reporting } \\
\text { institution }\end{array}$ & Report/Table & Frequency & $\begin{array}{l}\text { Submission } \\
\text { lag }\end{array}$ \\
\hline \multirow[t]{12}{*}{$\begin{array}{l}\text { I. Bank of } \\
\text { Uganda }\end{array}$} & Issuance of government securities. & Weekly & $\begin{array}{l}5 \text { working } \\
\text { days }\end{array}$ \\
\hline & Interest rates on government securities. & Weekly & $\begin{array}{l}5 \text { working } \\
\text { days }\end{array}$ \\
\hline & $\begin{array}{l}\text { Operations in the foreign exchange market and daily average } \\
\text { exchange rates. }\end{array}$ & Weekly & $\begin{array}{l}5 \text { working } \\
\text { days }\end{array}$ \\
\hline & Consumer price index. & Monthly & 2 weeks \\
\hline & $\begin{array}{l}\text { Balance sheet of the BOU, consolidated accounts of the } \\
\text { commercial banks, and monetary survey. The Internal Audit } \\
\text { Department (IAD) of the BOU will review the reconciliations } \\
\text { of monetary survey data with the financial records and the } \\
\text { audited financial statements. Any revisions to monetary } \\
\text { survey data, in line with the recommendations of the IMF } \\
\text { safeguards mission, will be documented and reconciled with } \\
\text { the previous presentation to ensure accurate reporting. }\end{array}$ & Monthly & 4 weeks \\
\hline & $\begin{array}{l}\text { Composition of foreign assets and liabilities of the BOU by } \\
\text { currency of denomination. }\end{array}$ & Monthly & 4 weeks \\
\hline & $\begin{array}{l}\text { Statement of (i) cash balances held in project accounts at } \\
\text { commercial banks; (ii) total value (measured at issue price) of } \\
\text { outstanding government securities from the Central } \\
\text { Depository System (CDS); and (iii) the stock of government } \\
\text { securities (measured at issue price) held by commercial banks } \\
\text { from the CDS. }\end{array}$ & Monthly & 6 weeks \\
\hline & $\begin{array}{l}\text { Summary of (i) monthly commodity and direction of trade } \\
\text { statistics; (ii) disbursements, principal and interest, flows of } \\
\text { debt rescheduling and debt cancellation, arrears, and } \\
\text { committed undisbursed balances-by creditor category; and } \\
\text { (iii) composition of nominal HIPC Initiative assistance, } \\
\text { disaggregated into grants, flow rescheduling, and stock-of- } \\
\text { debt reduction by creditor. }\end{array}$ & Monthly & 6 weeks \\
\hline & $\begin{array}{l}\text { Summary of stock of external debt, external arrears, and } \\
\text { committed undisbursed loan balances by creditor. }\end{array}$ & Quarterly & 6 weeks \\
\hline & $\begin{array}{l}\text { Standard off-site bank supervision indicators for deposit } \\
\text { money banks. }\end{array}$ & Quarterly & 4 weeks \\
\hline & $\begin{array}{l}\text { Summary table of preliminary program performance } \\
\text { comparing actual monthly outcome with adjusted program } \\
\text { targets for (i) base money; (ii) net claims on central } \\
\text { government by the banking system; (iii) stock of external } \\
\text { arrears; (iv) new nonconcessional external borrowing; and } \\
\text { (v) net international reserves }\end{array}$ & Quarterly & 5 weeks \\
\hline & $\begin{array}{l}\text { Daily average amount of commercial bank automatic access } \\
\text { to the BOU discount window and rediscounting of } \\
\text { government securities by commercial banks. }\end{array}$ & Quarterly & 4 weeks \\
\hline
\end{tabular}




\begin{tabular}{|c|c|c|c|}
\hline \multicolumn{4}{|c|}{ Table 1. Summary of Reporting Requirements (concluded) } \\
\hline $\begin{array}{l}\text { Reporting } \\
\text { institution }\end{array}$ & Report/Table & Frequency & $\begin{array}{l}\text { Submission } \\
\text { lag }\end{array}$ \\
\hline \multirow[t]{7}{*}{$\begin{array}{l}\text { II. Ministry of } \\
\text { Finance }\end{array}$} & $\begin{array}{l}\text { Summary of central government accounts. Revenues shall be } \\
\text { recorded on a cash basis. Expenditures shall be recorded when } \\
\text { checks are issued, except for domestic and external debt- } \\
\text { service payments, cash transfers to districts, and externally } \\
\text { funded development expenditures. Expenditures on domestic } \\
\text { interest will be recorded on an accrual basis and external debt } \\
\text { service will be recorded on a commitment basis (i.e., when } \\
\text { payment is due). Cash transfers to districts will be recorded as } \\
\text { expenditures of the central government when the transfer is } \\
\text { effected by the BOU. Expenditures on externally funded } \\
\text { development programs will be recorded as the sum of } \\
\text { estimated disbursements of project loans and grants by } \\
\text { donors, less the change in the stock of government project } \\
\text { accounts held at the BOU and domestic commercial banks. }\end{array}$ & Monthly & 6 weeks \\
\hline & $\begin{array}{l}\text { Summary of outstanding stock of group (B) domestic arrears. } \\
\text { Group (B) arrears comprise the stock of CCS/IFMS arrears } \\
\text { incurred after end-June } 2004 \text {. }\end{array}$ & Quarterly & 6 weeks \\
\hline & $\begin{array}{l}\text { Summary of contingent liabilities of the central government. } \\
\text { For the purpose of the program, contingent liabilities include } \\
\text { all borrowings by statutory bodies, government guarantees, } \\
\text { claims against the government in court cases that are pending, } \\
\text { or court awards that the government has appealed. }\end{array}$ & Quarterly & 6 weeks \\
\hline & $\begin{array}{l}\text { Detailed central government account of disbursed budget } \\
\text { support grants and loans, HIPC support, and external debt } \\
\text { service due and paid. }\end{array}$ & Monthly & 4 weeks \\
\hline & $\begin{array}{l}\text { Detailed central government account of disbursed donor } \\
\text { project support grants and loans. }\end{array}$ & Monthly & 6 weeks \\
\hline & $\begin{array}{l}\text { Statement on new loans contracted during the period } \\
\text { according to loan agreements. }\end{array}$ & Quarterly & 6 weeks \\
\hline & $\begin{array}{l}\text { Updated national accounts statistics (real and nominal) } \\
\text { according to UBOS and medium-term projections. }\end{array}$ & Quarterly & 4 weeks \\
\hline
\end{tabular}





\title{
INTERNATIONAL MONETARY FUND
}

UGANDA

\section{Third Review Under the Policy Support Instrument and Request for Waiver and Modification of Assessment Criteria-Informational Annex}

\author{
Prepared by the African Department \\ (In consultation with other departments) \\ Approved by Thomas Krueger and Mark Plant
}

June 25,2008

- Relations with the Fund. Describes financial and technical assistance by the IMF and provides information on the safeguards assessment and exchange system. Outstanding Fund credit was SDR 6 million (3 percent of quota) at end-April 2008.

- Joint Bank-Fund Work Program. Describes Bank-Fund collaboration through June 2009.

- $\quad$ Statistical Issues. Assesses the quality of statistical data. Economic data are generally adequate for surveillance purposes, although some shortcomings remain. 


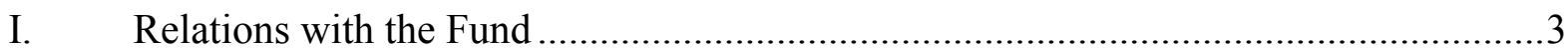

II. Joint Bank-Fund Work Program.....................................................................

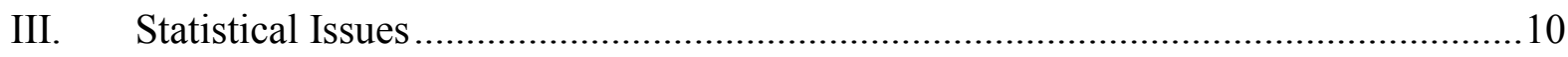




\section{APPENDIX I. Uganda: RELATIONS WITH THE FUND}

(As of April 30, 2008)

I. Membership Status: Joined 09/27/1963; Article VIII

\section{General Resources Account:}

Quota

Fund holdings of currency

III. SDR Department:

Net cumulative allocation

Holdings

\section{Outstanding Purchases and}

Loans:

Enhanced Structural Adjustment Facility

(ESAF)/Poverty Reduction and Growth

Facility (PRGF) arrangements

\section{Financial Arrangements:}

$\begin{array}{lcccc}\text { Type } & \begin{array}{c}\text { Approval } \\ \text { Date }\end{array} & \begin{array}{c}\text { Expiration } \\ \text { Date }\end{array} & \begin{array}{c}\text { Amount Approved } \\ \text { (SDR Million) }\end{array} & \begin{array}{c}\text { Amount Drawn } \\ \text { (SDR Million) }\end{array} \\ \text { PRGF } & 09 / 13 / 2002 & 01 / 31 / 2006 & 13.50 & 13.50 \\ \text { ESAF/PRGF } & 11 / 10 / 1997 & 03 / 31 / 2001 & 100.43 & 100.43 \\ \text { ESAF } & 09 / 06 / 1994 & 11 / 09 / 1997 & 120.51 & 120.51 \\ \text { ESAF } & 04 / 17 / 1989 & 06 / 30 / 1994 & 219.12 & 219.12\end{array}$

\author{
SDR Million \\ 180.50 \\ 180.51
}

SDR Million

29.40

0.28

SDR Million

6.00
$\%$ Allocation

100.00

0.95

$\%$ Quota

\section{Projected Payments to Fund}

(based on existing use of resources and present holdings of SDRs)

Principal

Forthcoming

Charges/Interest

Total
3.32

100.0

100.0

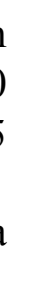

.


VII. Implementation of HIPC Initiative:

\begin{tabular}{|c|c|c|c|}
\hline & $\begin{array}{r}\text { Original } \\
\text { framework }\end{array}$ & $\begin{array}{r}\text { Enhanced } \\
\text { framework }\end{array}$ & Total \\
\hline \multicolumn{4}{|l|}{ Commitment of HIPC assistance } \\
\hline Decision point date & $4 / 23 / 1997$ & $2 / 7 / 2000$ & \\
\hline $\begin{array}{l}\text { Assistance committed (1999 NPV terms) }{ }^{1} \\
\text { Total assistance (US\$ million) }\end{array}$ & 347.00 & 656.00 & \\
\hline $\begin{array}{l}\text { Of which: Fund assistance } \\
\text { (US\$ million) }\end{array}$ & 68.90 & 91.00 & \\
\hline Completion point date & April 1998 & May 2000 & \\
\hline \multicolumn{4}{|l|}{ Delivery of Fund assistance (SDR million) } \\
\hline Assistance disbursed to the member & 51.51 & 68.10 & 119.61 \\
\hline Interim assistance & $\ldots$ & 8.20 & 8.20 \\
\hline Completion point balance & 51.51 & 59.90 & 111.41 \\
\hline Additional disbursement of interest income ${ }^{2}$ & & 2.06 & 2.06 \\
\hline
\end{tabular}

\section{Implementation of MDRI Assistance}

I. Total Debt Relief (SDR Million) ${ }^{3}$

87.73

Of which: MDRI

75.85

HIPC

II. Debt Relief by Facility (SDR Million)

\section{Delivery Date}

January 2006

\section{GRA}

N/A

Eligible Debt

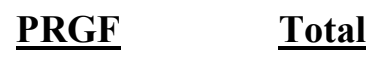

87.73

87.73

\footnotetext{
${ }^{1}$ Net present value (NPV) terms at the completion point under the original framework, and NPV terms at the decision point under the enhanced framework.

${ }^{2}$ Under the enhanced Initiative for Heavily Indebted Poor Countries (HIPC Initiative), an additional disbursement is made at the completion point corresponding to interest income earned on the amount committed at the decision point but not disbursed during the interim period.

${ }^{3}$ The Multilateral Debt Relief Initiative (MDRI) provides 100 percent debt relief to eligible member countries that are qualified for the assistance. The debt relief covers the full stock of debt owed to the Fund as of end-2004 which remains outstanding at the time the member qualifies for such debt relief. The MDRI is financed by bilateral contributions and the Fund's own resources, as well as the resources already disbursed to the member under the HIPC Initiative (see Section VII above).
} 


\section{Safeguards Assessments}

Under the Fund's safeguards policy, assessments with respect to the PSI are voluntary. An update assessment of the Bank of Uganda (BOU) was completed on April 10, 2007 and concluded that the BOU had strengthened its safeguards framework since the 2003 assessment. The main developments included implementation of International Financial Reporting Standards, publication of financial statements, establishment of an audit committee, and strengthening of the internal audit function. Staff made recommendations to address remaining vulnerabilities in the legal and internal control areas.

\section{Exchange Rate Arrangement}

The official exchange rate is determined on the interbank market for foreign exchange. As of December 31, 2007, the official exchange rate was U Sh 1,697 per U.S. dollar. The exchange system is free of restrictions on the making of payments and transfers for current international transactions. Uganda's exchange rate regime is classified as managed float without predetermined path.

\section{Article IV Consultation}

The Executive Board concluded the last Article IV consultation on December 15, 2006. The next Article IV consultation with Uganda will be held on the 24-month cycle, subject to the provisions of the decision on consultation cycles approved on July 15, 2002.

In February and April 2001, joint World Bank/Fund missions visited Kampala as part of the Financial Sector Assessment Program (FSAP). A final report was provided to the authorities in November 2001, and an MFD mission discussed the report with the authorities during the Article IV consultation discussion in November 2002, and the Financial System Stability Assessment was prepared for Board discussion in February 2003. In November 2004, a joint World Bank/Fund missions visited Kampala for a FSAP update.

\section{Policy Support Instrument (PSI)}

Uganda initiated a 16-month PSI with the Fund in January 2006. The 16-month PSI was replaced by a new three-year PSI in December 2006.

\section{Technical Assistance}

Uganda has received extensive technical assistance from the Fund in recent years. ${ }^{4}$

\footnotetext{
${ }^{4}$ For a description of technical assistance provided prior to 1998, see the staff report for Uganda's request for a three-year arrangement under the PRGF (Country Report 02/213).
} 
An FAD mission visited Kampala in April 1998 to advise the authorities on public service pension reform issues, and another mission visited Kampala in September 1998 to assist the authorities in improving customs administration procedures. A resident advisor in the area of local government budgeting began a six-month assignment in August 1998, which was subsequently extended to October 1999. An FAD resident advisor on budgeting and commitment control commenced a six-month assignment in November 1998, which was extended until June 2002. In October 2000, an FAD mission visited Kampala to provide technical assistance in tax policy and administration. A joint Bank/Fund TA mission visited Kampala in October 2003 to help harmonize tax investment incentives among the member countries of the EAC. An FAD TA mission on tax administration also visited Uganda in October 2004 to develop a comprehensive reform plan for the Uganda Revenue Authority (URA), followed by visits in April 2005 and April - May 2006. Two FAD resident advisors (coordinated through AFRITAC-East) assisted the implementation of a CCS at the local government level and the Fiscal Decentralization Strategy between June 2004 and February 2005. In the upcoming joint FAD-WB mission, the FAD will be represented by AFRITAC East, which would follow up on the recommendations of the 2005 FAD arrears mission and check whether the new financial and physical reporting system for local governments has been implemented.

A STA multisector statistics mission visited Kampala in December 1998 to conduct a comprehensive assessment of Uganda's macroeconomic statistics, including data compilation and dissemination, and to provide recommendations for improvements. Follow-up STA missions in national accounts and money and banking statistics visited Kampala in MarchApril 2000 to examine the status of implementation of the previous recommendations. A STA mission on government financial statistics (GFS) visited Uganda in December 2001 to assist authorities in improving fiscal reporting by establishing regular reporting systems that are aligned with the GFS Manual 2001, as well as ensuring consistency within monetary sector data for the government. A follow-up mission on GFS visited Uganda in May 2003. A mission visited Uganda during February-March 2002 to review balance of payment statistics and the progress in implementing the recommendations of the multisector mission of 1998 and of the national accounts mission of 2000 with respect to the measurement of goods imports. A follow-up mission on balance of payments statistics visited Uganda in August 2003. Uganda is participating in the General Data Dissemination System (GDDS) and its metadata were initially posted on the Fund's Dissemination Standards Bulletin Board in May 2000. A STA mission was in Uganda during February 2005 to prepare a data ROSC.

Since the 2001 FSAP, MFD's TA to Uganda has focused on liquidity management, exchange rate intervention, central bank accounting and auditing, and banking supervision. The authorities have effectively used TA advice on these topics, and they have made good use of an MFD resident advisor, who took up her initial appointment in mid-July 2001. The advisor was fully integrated into the supervision function and has been involved in all aspects of the work, including participating in on-site examinations; she has subsequently been replaced by a second resident advisor. MFD has fielded two TA missions (July 2001 and January 2002) to 
assist the authorities with liquidity management and address the problems of interest rate volatility and exchange rate interventions. The BOU has started implementing the recommendations made in the report, and now clearly separates sterilization operations and liquidity management; however, they are still having problems with interest rate and exchange rate volatility and have requested follow-up TA in this area. In March and SeptemberOctober 2002, MFD provided TA to improve central bank accounting and the Bank of Uganda's accounting manual. A TA evaluation visit was conducted in June 2003, and an MFD mission following up on monetary and exchange rate operations, public debt, and liquidity management was conducted in March 2004. An FSAP update mission was conducted in November 2004 and focused on access, outreach, and stability issues, which also updated progress made since the last FSAP. A TA mission on enhancing the effectiveness of monetary policy implementation and developing financial markets was conducted in August 2005. Finally, a joint MCM/BOU workshop on financial market development was held in April 2006.

\section{Future Technical Assistance Priorities}

The priorities for Fund technical assistance in the next few years will be in the areas of tax administration, public expenditure management, especially control and monitoring of public arrears at both central and local government levels, monetary and exchange rate management, bank supervision, national accounts statistics, reporting standards for government finance statistics, monetary and balance of payments statistical reporting, central bank accounting, and audit and debt management.

\section{Resident Representative}

The Fund has maintained a resident representative in Uganda since July 1982. 
APpendix II. Joint BANK-Fund WORK Program, July 2008 - June 2009

\begin{tabular}{|c|c|c|c|}
\hline Title & Products & $\begin{array}{l}\text { Provisional } \\
\text { timing of } \\
\text { missions (if } \\
\text { relevant) }\end{array}$ & $\begin{array}{l}\text { Expected } \\
\text { delivery } \\
\text { date }\end{array}$ \\
\hline \multicolumn{4}{|c|}{ A. Mutual information on relevant work programs } \\
\hline \multirow[t]{5}{*}{$\begin{array}{l}\text { Key elements } \\
\text { of World Bank } \\
\text { work program } \\
\text { in next } 12 \\
\text { months }\end{array}$} & $\begin{array}{l}\text { Poverty Reduction Support Credit to } \\
\text { enhance productivity and competitiveness, } \\
\text { effective delivery of social services, } \\
\text { public sector management, and human } \\
\text { development issues. }\end{array}$ & & June 2009 \\
\hline & $\begin{array}{l}\text { The work program will continue to } \\
\text { concentrate on areas within the existing } \\
\text { portfolio currently focusing on } \\
\text { infrastructure development (transport and } \\
\text { energy), local government service } \\
\text { delivery, agricultural research and } \\
\text { training, Kampala infrastructure } \\
\text { development, and local government } \\
\text { service delivery (including Northern } \\
\text { Uganda rehabilitation). In addition, the } \\
\text { Bank plans support for agricultural } \\
\text { technology and advisory services, } \\
\text { Northern Uganda social protection, roads } \\
\text { development, secondary education, } \\
\text { minerals development and environment. }\end{array}$ & & June 2009 \\
\hline & $\begin{array}{l}\text { Public Expenditure Review with the focus } \\
\text { on health sector and public sector wages. }\end{array}$ & & June 2009 \\
\hline & Study on fostering financial deepening & & June 2009 \\
\hline & $\begin{array}{l}\text { Study on "Understanding and Elimination } \\
\text { of Non-tariff barriers to support Trade } \\
\text { Integration with the EAC", }\end{array}$ & & June 2009 \\
\hline \multirow{3}{*}{$\begin{array}{l}\text { IMF work } \\
\text { program in } \\
\text { next } 12 \text { months }\end{array}$} & Article IV and Fourth Review under PSI & Sep.-Oct. 2008 & Dec. 2008 \\
\hline & Fifth Review under PSI & Mar.-Apr. 2009 & June 2009 \\
\hline & Staff visit on the government budget & May 2009 & June 2009 \\
\hline
\end{tabular}




\begin{tabular}{|c|c|c|c|}
\hline \multicolumn{4}{|c|}{ B. Requests for work program inputs } \\
\hline \multirow[t]{2}{*}{$\begin{array}{l}\text { Fund request to } \\
\text { Bank }\end{array}$} & $\begin{array}{l}\text { 1. Outline immediate priorities for } \\
\text { investment in infrastructure. }\end{array}$ & & Dec. 2008 \\
\hline & $\begin{array}{l}\text { 2. Clarify broad strategic priorities in the } \\
\text { PFM area; look at the composition of the } \\
\text { authorities' budget; and look at what can } \\
\text { be done to improve procurement } \\
\text { practices. }\end{array}$ & & Dec. 2008 \\
\hline \multirow[t]{2}{*}{$\begin{array}{l}\text { Bank request to } \\
\text { Fund }\end{array}$} & $\begin{array}{l}\text { 1. Monitoring of government contracting } \\
\text { of non-concessional borrowing. }\end{array}$ & Sep.-Oct. 2008 & Dec. 2008 \\
\hline & $\begin{array}{l}\text { 2. Monitoring of the cost of monetary } \\
\text { policy, and discussion on appropriate mix } \\
\text { of instruments with authorities. }\end{array}$ & Sep.-Oct. 2008 & Dec. 2008 \\
\hline \multicolumn{4}{|c|}{ C. Agreement on joint products and missions } \\
\hline $\begin{array}{l}\text { Joint products } \\
\text { in next } 12 \\
\text { months }\end{array}$ & $\begin{array}{l}\text { 1. Collaborate with the authorities on their } \\
\text { financial market development strategy } \\
\text { 2. Collaborate on the Joint DSA } \\
\text { 3. Collaborate on the JSAN }\end{array}$ & $\begin{array}{l}\text { Sep. - Oct. } \\
2008 \\
\text { Sep.- Oct. } 2008\end{array}$ & $\begin{array}{l}\text { Dec. } 2008 \\
\text { Dec. } 2008\end{array}$ \\
\hline
\end{tabular}




\section{APPENDIX III. UgANDA: STATISTICAL ISSUES}

Overall, data provision is adequate for surveillance purposes, although some shortcomings remain. Uganda participates in the General Data Dissemination System (GDDS) and its metadata were initially posted on the Fund's Dissemination Standards Bulletin Board in May 2000. Partial updates of real and external sector metadata were completed in August 2005. Uganda is participating in the SDDS, government finance, and the monetary and financial statistics modules of the Fund's GDDS Project for Anglophone Africa (funded by the U.K. Department for International Development (DFID)). This project aims to assist participating countries to implement plans for improvement identified in the metadata.

In February 2005 a STA mission prepared a data ROSC, with results published in July 2006. The ROSC mission assessed data compilation and dissemination practices against international standards in national accounts, prices, government finance, and balance of payments statistics; the monetary and financial statistics were not assessed. In April 2007 a mission conducted a Special Data Dissemination Standard (SDDS) assessment focusing on dissemination practices vis-à-vis the SDDS requirements for coverage, periodicity and timeliness.

\section{Real sector statistics}

Since 2004 Uganda has been receiving technical assistance from East AFRITAC on the compilation of quarterly national accounts and training through short missions and a regional workshop. Recently, compilers have developed estimates of quarterly value added at constant 2002 prices using the International Standard Industrial Classification (ISIC) Rev III groups. However, methodological shortcomings remain relating to benchmarking. The Uganda Bureau of Statistics (UBOS) collects output data from a sample of manufacturing business establishments in the industrial belt of the country to compile a Major and a Main Index of Industrial Production (IIP). Labor market indicators such as employment, unemployment, and wages/earnings are infrequently compiled and disseminated. UBOS aims to compile and disseminate these data categories on an annual basis, but due to resource and data unavailability, these data are compiled with a two year lag. UBOS collects prices of consumer goods and services from six urban centers for computing inflation rates, which are disseminated on the last working day of every month. According to UBOS, there are plans to expand the coverage to include another urban center. UBOS collects producer prices (factory gate prices) from a sample of manufacturing establishments, which undertake manufacturing activities as classified by ISIC. The price information is used to compute the Producer Price Index for Manufacturing (PPI-M).

\section{Government finance statistics}

The Ministry of Finance, Planning and Economic Development (MoFPED) compiles the fiscal statistics on general government including budgetary central government and local governments but excluding extra budgetary institutions and the National Social Security Fund. A revised chart of accounts was implemented for use by all budgetary central government and 
local government units from July 1, 2003. Uganda reports Government Finance Statistics (GFS) data according to the GFSM 2001 framework for the GFS Yearbook, but institutional coverage are limited to budgetary central government and local governments. External debt for the public sector, external debt guaranteed by the public sector, and debt service schedule data are disseminated in millions of dollars and in Uganda shillings (using end of year exchange rates) for the fiscal year (July to June). Uganda does not report any high frequency data for inclusion in the IFS. In terms of the DFID Phase II project, the authorities recently agreed that a GFS mission will visit Uganda in September 2008, to further assist with improving fiscal data.

\section{Monetary and financial statistics}

The monetary data are not currently updated in International Financial Statistic. The latest available data on the central bank are for January 2008 and on other depository corporations for September 2007.

In February 2006, a monetary and financial statistics mission found that there are a number of areas where improvements are needed to align the data with the recommendations of the Monetary and Financial Statistics Manual (MFSM). The detailed balance sheet of the Bank of Uganda (BOU) is well designed for producing the analytical data; however, (1) reverse repos between the BOU and the other depository corporations (ODCs) are not classified as advances to banks, (2) there is some inconsistency among departments in the BOU in determining which foreign assets are in reserve assets; (3) loans and advances are presented net of provisions for loans losses; (4) the proper classification of many positions of the Development Finance Department is difficult because the main classification used in the accounts is the original source of funds, rather than the unit subject to a claim or liability; and (5) the economic and accounting nature of administered and internally managed funds of the BOU is unclear.

The March 2008 monetary and financial statistics mission found that substantial progress was made in producing historical data on standardized report forms (SRF) that would align the monetary data in accordance with the MFSM. Four quarters of data on the new central bank's accounts were finalized in the 1SR format. Data were produced for commercial banks in the 2SR format for the period June 2006 - October 2007. Banks will begin reporting with data for May 2008 on the new system, which will run in parallel with the existing system for six months. The mission also recommended the consolidation of the credit institutions and microfinance deposit-taking institutions into the depository corporations survey and initiated the collection of data from insurance companies. No progress was made on savings and credit cooperatives.

\section{External sector statistics}

The 2005 data ROSC mission found that the balance of payments statistics broadly follow the fifth edition of the Balance of Payments Manual (BPM5), but that there are some departures from recommended definitions, scope, and classifications. The mission urged completion of 
the conversion to the BPM5 and the development of new source data and estimation techniques in the following areas: exports of freight and imports of passenger transportation, compensation of employees, direct investment abroad, portfolio investment and financial derivatives. International trade data could be improved by incorporation of results from the Survey of Informal Cross-Border Trade and greater use of trade partner country data sources.

Quarterly and fiscal year data are disseminated in the BOU's publications Quarterly Economic Report and Annual Report, as well as the UBOS annual Statistical Abstract. In principle, data cover the entire economy and include all transactions between residents and nonresidents. Quarterly balance of payments data are disseminated in an analytical format or on the basis of standard components, in accordance with the requirements of the IMF Balance of Payments Manual, Fifth Edition (BPM5).

A mission in April 2008 reviewed the private sector financial survey and an informal crossborder trade survey as well as estimation methods for remittances. Proposals were made for dealing with gaps in recording of transactions in the securities market by nonresidents through the secondary market and nominee accounts. It also proposed a priority program to cover developments in the oil industry and identified several alternative methods to improve the estimation of remittances. The mission also recommended a timely, small-scale quarterly survey of financial flows. 

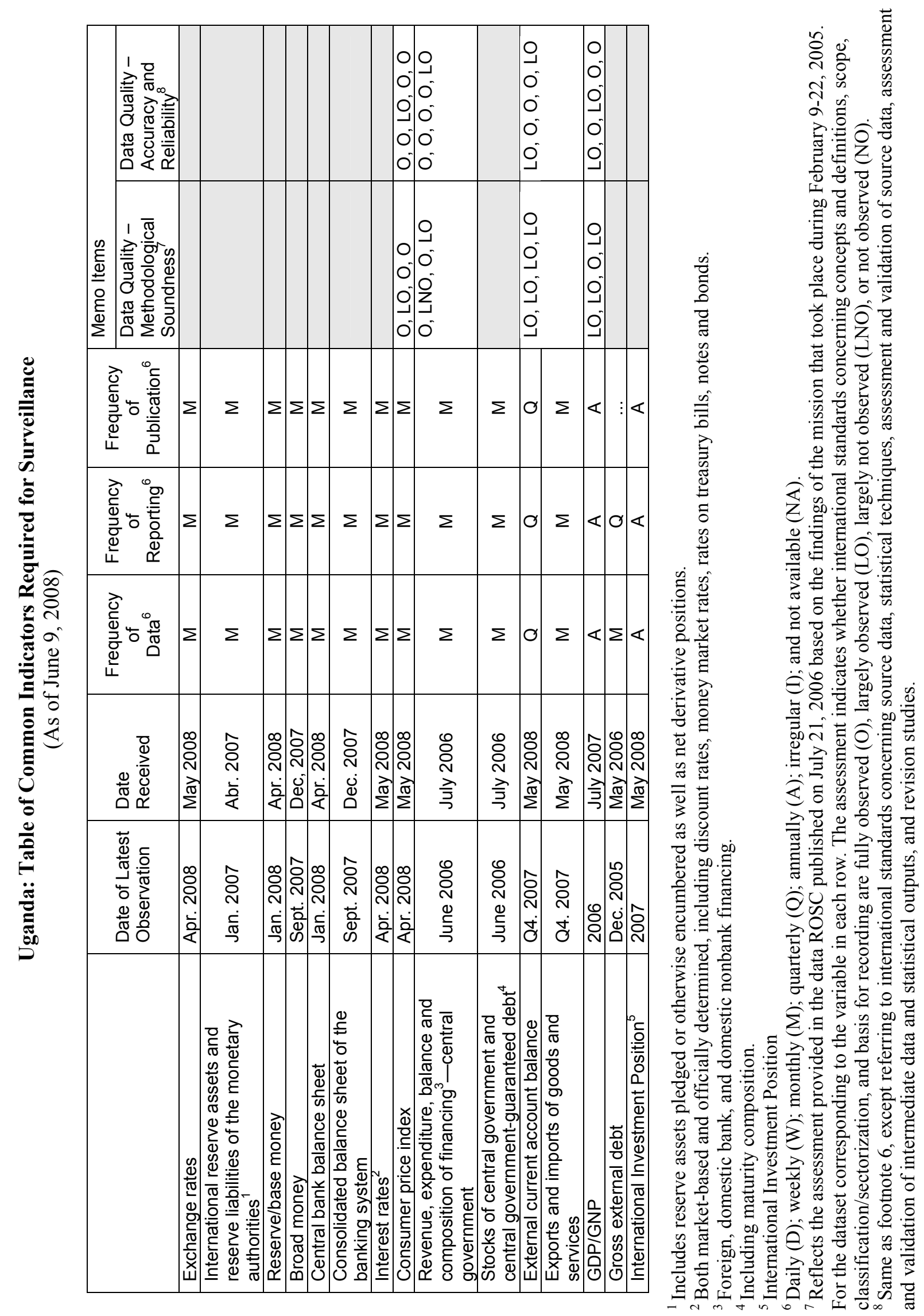

Press Release No. 08/172

FOR IMMEDIATE RELEASE

July 10, 2008
International Monetary Fund

Washington, D.C. 20431 USA

\section{IMF Executive Board Completes Third Review Under the Policy Support Instrument for Uganda}

The Executive Board of the International Monetary Fund (IMF) has completed the third review under a three-year Policy Support Instrument (PSI) for Uganda. The PSI was approved on December 15, 2006 (see Press Release No. 06/281). The program goals include macroeconomic stability, sustainable economic growth, poverty reduction, financial sector deepening, and improved public sector financial management.

In completing the third review under Uganda's PSI, the Executive Board approved Uganda's request for waivers of non-observance of four structural assessment criteria. The nonobservance of these four criteria was largely due to adjustments in light of new information and reassessment of costs and priorities. The changes are deemed not to constitute significant deviations from program goals. The Executive Board also approved a modification of the continuous assessment criterion on external debt and three end-June 2008 quantitative assessment criteria.

Following the Executive Board's discussion, Mr. Takatoshi Kato, Deputy Managing Director and Acting Chair, stated:

"Uganda's economy has continued to thrive, owing to appropriate fiscal and monetary policies, improved regional stability, and its resilience to shocks. The economic outlook remains positive, supported by higher public investment in infrastructure and sound economic policies. A comfortable level of foreign exchange reserves and sufficient locally-grown food supplies are helping Uganda cope with the sharp increases in world fuel and food prices.

"A continued focus on price stability as the primary objective of monetary policy remains essential. Rising food and fuel prices have lifted Uganda's inflation to about 11 percent. The authorities have expressed their commitment to prevent any spill over into sustained higher inflation, and are adjusting monetary policies accordingly for the coming year.

"Following strong fiscal consolidation in recent years, the authorities are well-placed to scale up domestic investment to alleviate pressing infrastructure bottlenecks. Careful evaluation of the planned projects in terms of their cost effectiveness and impact on debt sustainability, especially to the extent that financing is non-concessional, is warranted. The planned restraint on current 
expenditure, will need to be implemented in a manner that does not give rise to new budget arrears or disruptions to key public services.

"Strengthening the authorities' focus on the long-standing problem of domestic budget arrears is essential. While clearing of old arrears is proceeding apace, accumulation of new arrears has yet to be arrested. To this end, the authorities' proposed new measures to address technical shortcomings in public financial management and to strengthen internal compliance with budget spending limits are welcome," Mr. Kato said.

The IMF's framework for PSIs is designed for low-income countries (and small island states) that may not need, or want, IMF financial assistance, but still seek IMF advice, monitoring and endorsement of their policies. PSIs are voluntary and demand driven. PSI-supported programs are based on country-owned poverty reduction strategies adopted in a participatory process involving civil society and development partners and articulated in a Poverty Reduction Strategy Paper (PRSP). This is intended to ensure that PSI-supported programs are consistent with a comprehensive framework for macroeconomic, structural and social policies to foster growth and reduce poverty. Members' performance under a PSI is normally reviewed semi-annually, irrespective of the status of the program (see Public Information Notice No. 05/145). 


\section{Statement by Mr. Peter Gakunu, Executive Director for Uganda and Mr. Inyambo Mwanawina, Advisor to Executive Director}

July 7, 2008

My Ugandan authorities are appreciative of the support by Management, and thank staff for the valuable and constructive engagement with respect to the country's macroeconomic and structural reform program under the PSI. The authorities welcome the informative and balanced staff reports and are in broad agreement with the staff assessment.

\section{Recent economic developments}

The Ugandan economy continues to perform buoyantly as a result of a disciplined fiscal stance, implementation of prudent monetary policy, and strong effort in carrying out structural reforms. Real GDP growth was 7.4 percent in the FY 2006/07 and is projected at 8.9 percent in the FY 2007/08, propelled by expansions in services and manufacturing. The proportion of the people living below the poverty line has declined substantially. Underlying inflation, which edged upwards, was contained at 7.3 percent in 2006/07 and projected at 11.3 percent in 2007/08 against the program target of 8.1 percent and 4.8 percent, respectively. The impact of the political unrest in Kenya in December 2007 was somewhat prolonged with regard to upward pressure on prices, which, together with increased domestic and regional demand for goods and services, higher international oil and food prices and a sharp increase in electricity tariffs, contributed to the higher-than-expected inflation, which stood at 11.8 percent in May 2008. The balance of payments performance and capital inflows have been strong, resulting in modest appreciation pressures on the Uganda shilling against the US dollar. Foreign exchange reserves equivalent to 5.8 months of import cover in $2006 / 07$ are projected to increase to 6.0 months of imports in 2007/08.

The fiscal performance to date and the outlook for the remainder of FY 2007/08 is in line with the Medium-Term Expenditure Framework (MTEF) and program objectives set out in the Memorandum of Economic and Financial Policies (MEFP). The better than expected tax collection in the first half of 2007/08 more than offset the negative impact on customs revenue collection from the unrest in Kenya. Overall, revenues are expected to perform better than targeted. Spending was within the budget envelope despite expenditure pressures arising from hosting the Commonwealth Heads of Government Meeting and the response to emergencies (floods in the North and East, and Ebola outbreak in the West) which were offset by expenditure adjustments in non-priority areas. The lower than expected levels of government implementation capacity, largely contributed to a budget surplus for the first half of $2007 / 08$.

The authorities recognize and remain committed to resolving a number of challenges in order to ensure sustained higher growth performance. These challenges include, expanding the 
fiscal space to finance domestic infrastructure investment and increased spending pressure arising from reconstruction of Northern Uganda, prudent fiscal management, stemming the growth of domestic arrears, tightening monetary policy in order to bring inflation down to less than 5 percent, prudent management of large foreign exchange inflows, removal of structural rigidities in the financial sector, and managing the macroeconomic consequences of fast rising oil prices.

\section{Performance under the PSI}

The authorities remain committed to maintaining macroeconomic stability and implementing structural reforms, which has resulted in all the assessment criteria through end-December 2007 being met. In addition, performance in the first half of 2007/08 was broadly satisfactory, although the indicative target for poverty related spending was not observed because of delays in recruitment of teachers and compliance by spending agencies, even though the required resources were made available. On the structural side, the debt strategy was published in December 2007 and most of the other structural measures were on track. However, four structural assessment criteria for May and June 2008 were missed--delay in issuance of the tender for the national identity card system; establishment of a single regulatory body for non-bank financial sectors for which the authorities opted for specialized regulatory agencies; slight delay in the roll over of the new Integrated Personnel and Payroll System; and budgetary allocation towards the reduction of the stock of domestic arrears. The nonobservance of these structural criteria was mainly on account of improvement in program design in the light of new information, as elaborated in the staff report.

\section{Medium-term outlook and policy objectives}

The central objective of the authorities is to raise real economic growth to an average of 8 percent per annum in the medium-term, supported by sound public finances and prudent monetary policy in order to ensure job creation and 'Prosperity For All'. The focus is to remove constraints affecting private investment while the prevailing peace in Northern Uganda, strong domestic and regional market, as well as peace and stability in the East and Central African regions provide further impetus to growth. 


\section{Fiscal policy}

The fiscal policy objectives are set in the MEFP and the MTEF. The authorities recognize the need to restrain expenditure and improve revenue collection by 0.5 percent of GDP per year in line with the MTEF for the remainder of FY2007/08 and FY 2008/09. The authorities are committed to taking measures to improve the implementation capacity, including the creation of the Uganda Roads Authority. A comprehensive audit review of the IFMS is to be completed in September 2008 and expenditures contracted outside the IFMS will not be recognized by the Accountant General with effect from July 2008. The authorities are committed to exercising discipline in contracting new loans to maintain debt sustainability. Priority in spending has been directed towards agriculture, infrastructure (energy, roads and railways) human resource development, environment for private sector development-including construction of four industrial parks, and provision of tax incentives to qualifying investors in export business.

The authorities recognize that much more needs to be done to facilitate the flow of commodities in and out of the country in the wake of the recent Kenyan crisis and the increased regional demand for Ugandan goods and services. An additional US\$200 million per year for the next 5 years has been budgeted and ring-fenced to upgrade key transport corridors linking the country to Southern Sudan, Eastern DRC, Kenya and Rwanda, while discussions with Tanzania are envisaged to open a sea route through Dar-es-salaam as a viable alternative. The Uganda-Kenya concession agreement for the Rift Valley Railway line is being reviewed. The Bujagali hydroelectric dam and power plant has been under construction for one year and Karuma hydro power project is in the pipeline.

\section{Monetary policy and financial sector}

Monetary policy aims at maintaining annual average underlying inflation below 5 percent within the framework of the reserve money target for both FY 2007/08 and 2008/09. The authorities are committed to manage liquidity using an appropriate policy instrument mix without exacerbating market instability and loss of export competitiveness. Sterilization of excess liquidity will continue to rely on both indirect monetary policy instruments and foreign exchange sales. The mix of instruments will be kept under review due to persistent capital inflows to avoid undue pressure on interest and exchange rates. The authorities realize that maintaining low and stable single-digit inflation, stemming financial market instability and minimizing export competitiveness losses, against the back drop of high internal food and oil prices, is a delicate balancing act. They recognize that enhancing the efficiency and productivity of the export sector is key to sustaining the high export growth momentum. Deposit auctions have been introduced to expand the array of monetary policy instruments for liquidity management. The cost of sterilization is being shared between the fiscal and monetary authorities.

The authorities are still reviewing preconditions for, and appropriateness of, an inflation targeting framework in the medium-term. In the meantime, they are continuing with a 
flexible reserve money growth target range, to take into account shifts in both the velocity and the money multiplier, and the surge in net capital inflows. The base money growth target has been adjusted further upwards by 3 percentage points in view of higher economic growth and increased money demand. Furthermore, the authorities recognize that the high cost of borrowing for small scale enterprises is a serious impediment to attaining higher economic growth performance, employment creation and poverty reduction. In this regard, the authorities are committed to enhancing the development of the financial sector. New commercial banks have been allowed entry to boost competition in the sector. The Uganda Development Bank (UDB) is gradually being recapitalized to ensure availability of long-term financing, which is to be based on internationally accepted best practices. A credit guarantee scheme to cover up to 50 percent of credit risk associated with loans by commercial banks for certain sectors will be introduced.

\section{Structural reforms}

The authorities are committed to sustaining the implementation of the country's economic reform program under the PSI and PEAP. New bodies to regulate nonbank financial institutions (except UDB) are being established. The existing Insurance Commission will continue to regulate the insurance industry. A draft legislation for establishing a regulator for the pension sector and finding an appropriate entity to supervise the other non deposit taking financial service providers, such as leasing companies and microfinance institutions, are underway. The authorities recognize the need to supervise the UDB, which will be done by the newly reconstituted and independent board for the time being. The issuance of a financial score cards and envisaged establishment of the Credit Reference Bureau (CRB) are aimed not only at reducing identity fraud-related losses but also information asymmetry between banks and borrowers.

Measures aimed at improving the efficiency of tax tribunal and its legal capacity to handle cases are being undertaken. The authorities are also considering introducing an electronic tax registry. The BoU, in consultation with the Monetary Affairs Committee of the East African Community, has drafted a comprehensive financial market development plan aimed at enhancing the effectiveness and efficiency of the financial sector, including financial deepening.

The authorities are also updating the national energy policy to ensure that oil revenue is managed and invested prudently, in an accountable manner and transparently. An agreement has been reached on the principles of operating the energy fund.

The introduction of a national identity card system has taken longer than previously envisaged due to high cost but the authorities are taking some interim measures to enhance existing databases.

\section{Conclusion}

The authorities have remained steadfast in their commitment to implementing prudent macroeconomic and structural policies under the PSI. All the assessment criteria through 
end-December 2007 were observed but one structural assessment criterion for end-May 2008 was not met - implementation of a pilot Integrated Personnel and Payroll System in three commissions, four ministries and local governments--and three structural assessment criteria for end-June 2008 were missed - submission to cabinet of a policy paper outlining the establishment of a new regulatory framework for financial institutions, U Sh300 billion for payment of domestic arrears, and issuance of a tender for national identity card system - as a consequence of new information and reassessment of costs and priorities. Accordingly, the authorities are seeking waivers in view of the remedial measures that have been put in place. The authorities believe that the policies set out in the MEFP are adequate to achieve the objectives of their PSI-supported program. They are committed to working with Fund and other development partners in the implementation of the program. The authorities, therefore, request for the completion of the third review under the PSI. 